\title{
Dynamics of Eddy-Driven Low-Frequency Dipole Modes. Part I: A Simple Model of North Atlantic Oscillations
}

\author{
Dehai Luo \\ Physical Oceanography Laboratory, College of Physical and Environmental Oceanography, Ocean University of China, \\ Qingdao, China \\ ANTHONY R. LuPO \\ Department of Soil, Environmental and Atmospheric Sciences, University of Missouri-Columbia, Columbia, Missouri \\ HAN WAN \\ College of Physical and Environmental Oceanography, Ocean University of China, Qingdao, China
}

(Manuscript received 28 November 2005, in final form 12 April 2006)

\begin{abstract}
A simple theoretical model is proposed to clarify how synoptic-scale waves drive the life cycle of the North Atlantic Oscillation (NAO) with a period of nearly two weeks. This model is able to elucidate what determines the phase of the NAO and an analytical solution is presented to indicate a high similarity between the dynamical processes of the NAO and zonal index, which is not derived analytically in previous theoretical studies. It is suggested theoretically that the NAO is indeed a nonlinear initial-value problem, which is forced by both preexisting planetary-scale and synoptic-scale waves. The eddy forcing arising from the preexisting synoptic-scale waves is shown to be crucial for the growth and decay of the NAO, but the preexisting low-over-high (high-over-low) dipole planetary-scale wave must be required to match the preexisting positive-over-negative (negative-over-positive) dipole eddy forcing so as to excite a positive (negative) phase NAO event. The positive and negative feedbacks of the preexisting dipole eddy forcing depending upon the background westerly wind seem to dominate the life cycle of the NAO and its life period.

An important finding in the theoretical model is that negative-phase NAO events could be excited repeatedly after the first event has decayed, but for the positive phase downstream isolated dipole blocks could be produced after the first event has decayed. This is supported by observed cases of the NAO events presented in this paper. In addition, a statistical study of the relationship between the phase of the NAO and blocking activity over Europe in terms of the seasonal mean NAO index shows that blocking events over Europe are more frequent and long-lived for strong positive-phase NAO years, indicating that the positivephase NAO favors the occurrence of European blocking events.
\end{abstract}

\section{Introduction}

In recent years, the North Atlantic Oscillation (NAO), a zonally localized, north-south dipole mode of atmospheric low-frequency variability in the Northern Hemisphere $(\mathrm{NH})$ over the North Atlantic sector, has received great attention in the literature. The NAO is not only related to European weather and climate,

Corresponding author address: Dr. Dehai Luo, College of Physical and Environmental Oceanography, Ocean University of China, Qingdao 266003, China.

E-mail: 1dh@ouc.edu.cn but could also be impacted by global warming (Thompson and Wallace 2000; Thompson et al. 2000) or by stratospheric and oceanic changes (Visbeck et al. 2001). Although the surface (ocean and land), stratospheric, or even anthropogenic processes can influence the phase and amplitude of the NAO on time scales of a season or longer (Marshall et al. 2001; Wu and Gordon 2002; Wu and Liu 2005), the NAO, with its fundamental time scale of less than one month, is thought to be a natural mode of atmospheric variability (Christoph et al. 2000; Feldstein 2003; Jin et al. 2006a,b).

Many studies have suggested that for both phases, the synoptic-scale waves are a dynamical driving

DOI: $10.1175 / J A S 3818.1$ 
mechanism of the NAO occurrence (Hurrell 1995; Feldstein 2003; Vallis et al. 2004; Benedict et al. 2004; Franzke et al. 2004; Gerber and Vallis 2005). Recently, Feldstein (2003) emphasized that the NAO life cycle within a period of about two weeks is fundamentally a nonlinear process and is driven by both high frequency and low frequency transient eddy vorticity fluxes. NAO decay, however, is due to the combined influence of Ekman pumping and the low-frequency transient eddy vorticity fluxes. The synoptic study of Benedict et al. (2004) showed that throughout NAO events, for both phases, the NAO is excited and maintained by the successive breaking of upstream synoptic-scale transients, consistent with the numerical simulations of Franzke et al. (2004).

In Vallis et al. (2004), a barotropic model was employed to illustrate a dynamical mechanism for the generation of meridional dipole structures similar to the Northern annular modes (NAM) and NAO-like patterns. They found that a large-scale midlatitude stirring provided by baroclinic synoptic-scale eddies in one region and represented by a simple stochastic forcing can excite an NAO-like mode with time scales of 10-50 days. Benedict et al. (2004) and Franzke et al. (2004) suggested that the NAO can be interpreted as a nonlinear initial-value problem. Thus, whether the NAO can be excited by synoptic-scale eddies depends, to some extent, upon the structures of the preexisting planetary-scale wave and synoptic- scale eddies. However, how a preexisting planetary-scale wave evolves into a NAO-like mode under the forcing of synopticscale eddies is still unresolved theoretically. It is also unclear what determines the phase of the NAO. In particular, no analytical solution has been derived in order to account for the close similarity between the dynamical processes of the NAO and zonal index. The purpose of this paper is to establish a theoretical model to elucidate the basic issues noted above.

This paper is organized as follows: in section 2, we establish a weakly nonlinear model to clarify the basic physics of the life cycle of eddy-driven low-frequency dipole modes such as NAO events by extending the theoretical model of blocking dynamics proposed in Luo (2005a). This is possible since there is a close similarity between blocking events and negative-phase NAO $\left(\mathrm{NAO}^{-}\right.$) events (Benedict et al. 2004). In section 3 , the dynamic mechanism for the onset of $\mathrm{NAO}^{-}$ events is explored through this theoretical model. Section 4 presents the dynamic process of synoptic-scale waves involved in the life cycle of positive-phase NAO $\left(\mathrm{NAO}^{+}\right)$events. We will also demonstrate that localized dipole blocks can occur downstream of the region of the $\mathrm{NAO}^{+}$event after the initial positive-phase event has decayed. In section 5, two case studies of NAO events are presented to indicate that European blocking can occur after an $\mathrm{NAO}^{+}$event decays, but this tendency is unlikely to be observed for $\mathrm{NAO}^{-}$ events. In addition, a statistical study of winter European blocking events during strong $\mathrm{NAO}^{-}$and $\mathrm{NAO}^{+}$ years (classified using the seasonal mean NAO index) shows that more frequent and long-lived blocking events can occur over Europe during $\mathrm{NAO}^{+}$years. The similarity between the zonal index and the NAO life cycle for dynamic processes is demonstrated theoretically using the analytical solutions derived in section 6 . Section 7 presents the discussion and conclusions.

\section{Evolution equation of eddy-driven NAO anomaly}

\section{a. The model}

Here, we try to establish a weakly nonlinear model to describe the life cycle of the NAO event associated with synoptic-scale waves even though the NAO event is probably a strongly nonlinear process. Because the NAO anomaly usually exhibits a barotropic dipole structure (Feldstein 2003; Vallis et al. 2004), a nondimensional equivalent barotropic model similar to that used in Luo (2005a), but without topography, will be used in the present paper.

To simplify the problem, the background westerly wind is assumed to be uniform. The scale separation assumption proposed in Luo (2005a) is used to analytically investigate the interaction between planetaryscale and synoptic-scale waves leading to the onset of low-frequency dipole modes such as NAO events.

If the atmospheric streamfunction, nondimensionalized with the velocity scale $U$ and length scale $L$, is decomposed into three parts: the background state field ( $\psi_{0}=-u_{0} y$, where $u_{0}$ is a uniform westerly wind), planetary-scale $(\psi)$, and synoptic-scale $\left(\psi^{\prime}\right)$ waves. Then, under this scale partitioning, the planetary-to-synopticscale interaction equations in a uniform background flow can be obtained as (Luo 2005a)

$$
\begin{gathered}
\left(\frac{\partial}{\partial t}+u_{0} \frac{\partial}{\partial x}\right)\left(\nabla^{2} \psi-F \psi\right)+J\left(\psi, \nabla^{2} \psi\right)+\left(\beta+F u_{0}\right) \frac{\partial \psi}{\partial x} \\
=-J\left(\psi^{\prime}, \nabla^{2} \psi^{\prime}\right)_{P} \\
\left(\frac{\partial}{\partial t}+u_{0} \frac{\partial}{\partial x}\right)\left(\nabla^{2} \psi^{\prime}-F \psi^{\prime}\right)+\left(\beta+F u_{0}\right) \frac{\partial \psi^{\prime}}{\partial x} \\
=-J\left(\psi^{\prime}, \nabla^{2} \psi\right)-J\left(\psi, \nabla^{2} \psi^{\prime}\right)+\nabla^{2} \psi_{S}^{*}
\end{gathered}
$$


where the subscript " $P$ " denotes a planetary-scale projection, which is used to represent the planetary-scale component of $J\left(\psi^{\prime}, \nabla^{2} \psi^{\prime}\right)$. The variable $\psi_{S}^{*}$ is a timedependent synoptic-scale vorticity source term that is introduced to maintain preexisting synoptic-scale waves, $\beta$ is the nondimensional meridional gradient of the Coriolis parameter, and $F=\left(L / R_{d}\right)^{2}$, where $R_{d}$ is the Rossby radius of deformation, and the boundary conditions used are the same as those in Luo (2005a).

It should be pointed out that, since the planetaryscale wave and synoptic-scale eddies having a narrow wavenumber range are assumed to be zonally separated as in Luo (2005a), $J\left(\psi, \nabla^{2} \psi^{\prime}\right)$ and $J\left(\psi^{\prime}, \nabla^{2} \psi\right)$ are within a synoptic-scale range. In this case, they only appear in synoptic-scale equation (1b). Moreover, $-J\left(\psi^{\prime}, \nabla^{2} \psi^{\prime}\right)_{S}$ can be neglected in (1b) in that it lies remarkably outside the synoptic-scale range. In (1a), the term $-J\left(\psi^{\prime}\right.$, $\left.\nabla^{2} \psi^{\prime}\right)_{P}$ is the planetary-scale component of the nonlinear self-interaction of preexisting synoptic-scale eddies and is referred to as preexisting eddy forcing (PEF). Also there is $-J\left(\psi^{\prime}, \nabla^{2} \psi^{\prime}\right)_{P}=-\boldsymbol{\nabla} \cdot\left(\mathbf{V} q^{\prime}\right)_{P}$ if

$$
\mathbf{V}^{\prime}=-\frac{\partial \psi^{\prime}}{\partial y} \mathbf{i}+\frac{\partial \psi^{\prime}}{\partial x} \mathbf{j}
$$

and $q^{\prime}=\nabla^{2} \psi^{\prime}-F \psi^{\prime}$ are defined for the unit vectors $\mathbf{i}$ and $\mathbf{j}$ in the $x$ and $y$ directions. In the diagnostic study of Feldstein (2003), the defined high-frequency transient eddy fluxes correspond in fact to a time-averaged term $-\boldsymbol{\nabla} \cdot \overline{\left(\mathbf{V}^{\prime} q^{\prime}\right)}$, which is a low-frequency component and similar in form to term $-J\left(\psi^{\prime}, \nabla^{2} \psi^{\prime}\right)_{P}$. However, it must be pointed out that, if a time-averaged equation is used in (1a) as in previous models, understanding how the planetary wave evolves into a NAO-like mode in the presence of synoptic-scale eddies becomes rather difficult. This problem can be avoided if the planetaryscale projection $-J\left(\psi^{\prime}, \nabla^{2} \psi^{\prime}\right)_{P}$ is used in (1a).

In the midhigh latitudes, $-J\left(\psi^{\prime}, \nabla^{2} \psi^{\prime}\right)_{P}$ inevitably has a dipole meridional structure in that the synopticscale eddies have, in general, a monopole meridional structure (Frederiksen 1982). In this case, the eddydriven low-frequency modes should have a dipole meridional structure. This means that the model that we establish here applies to low-frequency dipole modes like dipole blocking events, NAO, NAM, or even the Arctic Oscillation (AO). However, if synoptic-scale eddies comprise dipole and monopole meridional structures, this theoretical model can probably be extended to examine the dynamics of the well-known PacificNorth America (PNA) patterns, but this is beyond the scope of this paper. The emphasis of this paper is focused on the dynamics of the NAO because the NAO and $\mathrm{AO}$ are similar in this respect (Wallace 2000).

\section{b. Derivation of eddy-driven NAO anomaly evolution equation}

As stated in the introduction, many studies have revealed that the NAO occurrence is primarily attributed to synoptic-scale eddies (Hurrell 1995; Feldstein 2003; Vallis et al. 2004; Benedict et al. 2004; Franzke et al. 2004). However, most NAO studies are focused upon the observational and numerical aspects. Recently, Feldstein (2003) noted that the NAO life cycle is dominated by nonlinear processes. This motivates us to investigate how the synoptic-scale eddies reinforce the NAO life cycle and respond to it in a weakly nonlinear framework even though the NAO processes may sometimes occur within a strongly nonlinear regime. In fact, it is difficult to analytically investigate the relationship between an NAO anomaly and synoptic-scale eddies unless some assumptions are made.

Here, we extend the weakly nonlinear asymptotic theory proposed in Luo (2005a) to examine the physical dynamics of eddy-driven NAO events in that the composite field of $\mathrm{NAO}^{-}$events show a typical pattern resembling that of blocking flow (Benedict et al. 2004, their Fig. 5). It is also useful to assume; $\psi=\varepsilon \tilde{\psi}, \psi^{\prime}=$ $\varepsilon^{3 / 2} \tilde{\psi}^{\prime}$, and $\psi_{S}^{*}=\varepsilon^{5 / 2} \psi_{S}^{\prime}$ for a small parameter $\varepsilon$ in order to obtain the evolution equation of the NAO anomaly, as done in Luo (2005a), even though this assumption may be inconsistent with some observational studies that synoptic-scale eddies sometimes have almost the same amplitude as the NAO anomaly (Benedict et al. 2004). The advantage of this theoretical model, however, is that it is able to shed insight on the underlying dynamics of eddy-driven NAO events because the analytical solutions of the eddy-driven NAO anomaly and associated synoptic-scale eddies can be derived. Of course, our weakly nonlinear model can be considered as the basis for a complete theory in the future.

Using the above assumptions, Eqs. (1a)-(1b) can be rewritten as

$$
\begin{gathered}
\left(\frac{\partial}{\partial t}+u_{0} \frac{\partial}{\partial x}\right)\left(\nabla^{2} \tilde{\psi}-F \tilde{\psi}\right)+\varepsilon J\left(\tilde{\psi}, \nabla^{2} \tilde{\psi}\right)+\left(\beta+F u_{0}\right) \frac{\partial \tilde{\psi}}{\partial x} \\
=-\varepsilon^{2} J\left(\tilde{\psi}^{\prime}, \nabla^{2} \tilde{\psi}^{\prime}\right)_{P}, \\
\left(\frac{\partial}{\partial t}+u_{0} \frac{\partial}{\partial x}\right)\left(\nabla^{2} \tilde{\psi}^{\prime}-F \tilde{\psi}^{\prime}\right)+\left(\beta+F u_{0}\right) \frac{\partial \tilde{\psi}^{\prime}}{\partial x} \\
=-\varepsilon J\left(\tilde{\psi}^{\prime}, \nabla^{2} \tilde{\psi}\right)-\varepsilon J\left(\tilde{\psi}, \nabla^{2} \tilde{\psi}^{\prime}\right)+\varepsilon \nabla^{2} \psi_{s}^{\prime} .
\end{gathered}
$$

The evolution equation of the eddy-driven NAO anomaly can be derived from (2a)-(2b) by introducing 
slow time and space scales $T_{1}=\varepsilon t, T_{2}=\varepsilon^{2} t, X_{1}=\varepsilon x$, and $X_{2}=\varepsilon^{2} x$ (Luo 2005a).

If the fields $\tilde{\psi}$ and $\tilde{\psi}^{\prime}$ are expanded as

$$
\begin{aligned}
\tilde{\psi} & =\sum_{n=1}^{\infty} \varepsilon^{n-1} \tilde{\psi}_{n-1}\left(x, y, t, T_{1}, T_{2}, X_{1}, X_{2}\right) \\
\tilde{\psi}^{\prime} & =\tilde{\psi}_{0}^{\prime}\left(x, y, t, X_{1}\right)+\sum_{n=1}^{\infty} \varepsilon^{n} \tilde{\psi}_{n}^{\prime}\left(x, y, t, T_{1}, T_{2}, X_{1}, X_{2}\right)
\end{aligned}
$$

then the perturbation equations of planetary-scale and synoptic-scale waves can be obtained. In (3b), the inclusion of the scale $X_{1}$ in $\tilde{\psi}_{0}^{\prime}$ is to fix the position of the preexisting synoptic-scale eddies relative to the region where the NAO anomaly develops downstream (Feldstein 2003; Benedict et al. 2004) even though the formation of NAO anomalies is not known.

For the weakly nonlinear theory considered here, both $\tilde{\psi}_{0}$ and $\tilde{\psi}_{0}^{\prime}$ satisfy the linear Rossby wave equations (Luo 2005a). It can be noted that for the order series in (3),

$$
\begin{aligned}
\frac{\varepsilon^{n+1} \tilde{\psi}_{n+1}}{\varepsilon^{n} \tilde{\psi}_{n}} & =\varepsilon \frac{\tilde{\psi}_{n+1}}{\tilde{\psi}_{n}} ; \quad \frac{\varepsilon^{n+1} \tilde{\psi}_{n+1}^{\prime}}{\varepsilon^{n} \tilde{\psi}_{n}^{\prime}}=\varepsilon \frac{\tilde{\psi}_{n+1}^{\prime}}{\tilde{\psi}_{n}^{\prime}} \\
\frac{\tilde{\psi}_{n+1}}{\tilde{\psi}_{n}} & =O(1) ; \quad \frac{\tilde{\psi}_{n+1}^{\prime}}{\tilde{\psi}_{n}^{\prime}}=O(1)
\end{aligned}
$$

are necessarily satisfied in (3a)-(3b) to allow Eqs. (2a)(2b) to have asymptotic solutions in a weakly nonlinear theory. This does not imply that $\tilde{\psi}_{0}=O(1), \varepsilon \tilde{\psi}_{1}=O(\varepsilon)$, $\tilde{\psi}_{0}^{\prime}=O(1)$, and $\varepsilon \tilde{\psi}_{1}^{\prime}=O(\varepsilon)$ must be required in (3a)(3b). In the real application, the perturbation expansions are also correct even if the second-order term is the same as or even larger than the first-order term. Otherwise, weakly nonlinear theory cannot be applied to the dynamics of large-amplitude large-scale anomalies such as blockings (Haines and Malanotte-Rizzoli 1991). Thus, this weakly nonlinear limit is now removed in the application of the weakly nonlinear theory here.

Observations show that the NAO anomaly exhibits a typical dipole pattern, consisting of one center near southern Greenland and another broad center across the midlatitude North Atlantic (Hurrell 1995; Wallace 2000; Feldstein 2003). But the preexisting synopticscale eddies have a monopole meridional structure before they interact with the NAO anomaly. Thus, it is natural that the solutions of the leading order approximations $\tilde{\psi}_{0}$ and $\tilde{\psi}_{0}^{\prime}$ in (3a)-(3b) can be sought to be of the form

$$
\begin{aligned}
\tilde{\psi}_{0}= & A\left(T_{1}, T_{2}, X_{1}, X_{2}\right) \sqrt{\frac{2}{L_{y}}} \exp [i(k x-\omega t)] \sin (m y) \\
& + \text { c.c., } \\
\tilde{\psi}_{0}^{\prime}= & f_{0}^{\prime}\left(X_{1}\right)\left\{\exp \left[i\left(\tilde{k}_{1} x-\tilde{\omega}_{1} t\right)\right]\right. \\
& \left.+\alpha \exp \left[i\left(\tilde{k}_{2} x-\tilde{\omega}_{2} t\right)\right]\right\} \sin \left(\frac{m}{2} y\right)+\text { c.c., }
\end{aligned}
$$

where

$$
\begin{aligned}
& \omega=u_{0} k-\frac{\left(\beta+F u_{0}\right) k}{k^{2}+m^{2}+F}, \quad k=n k_{0}, \\
& m= \pm 2 \pi / L_{y} ; \quad \tilde{\omega}_{i}=u_{0} \tilde{k}_{i}-\frac{\left(\beta+F u_{0}\right) \tilde{k}_{i}}{\tilde{k}_{i}^{2}+m^{2} / 4+F} \\
& (i=1,2) ; \quad \tilde{k}_{1}=(\tilde{n}-\Delta \tilde{n}) k_{0}, \\
& \tilde{k}_{2}=(\tilde{n}+\Delta \tilde{n}) k_{0}, \quad k_{0}=\frac{1}{6.371 \cos \left(\phi_{0}\right)} ;
\end{aligned}
$$

$f_{0}^{\prime}=a_{0}^{\prime} \exp \left[-\mu\left(X_{1}+\varepsilon x_{0}\right)^{2}\right]$ for $\mu>0, \alpha= \pm 1 ; 2 \Delta \tilde{n} k_{0}$ is the difference between $\tilde{k}_{1}$ and $\tilde{k}_{2} ; \phi_{0}$ is the latitude; $L_{y}$ is the width of the beta channel; $x_{0}$ is the position of the maximum amplitude of the synoptic eddies relative to the preexisting planetary wave prior to the NAO onset; $a_{0}^{\prime}$ denotes the amplitude of preexisting synoptic-scale eddies; c.c. denotes the conjugate of its preceding term; and $A$ is the slowly varying complex amplitude of the dipole Rossby mode. In this study, if $\Delta \tilde{n} \sim 1$ and $n=2$ are allowed, there is $\tilde{k}_{2}-\tilde{k}_{1} \sim k$ and $\tilde{\psi}_{0}^{\prime}$ in (3b) characterizes synoptic-scale waves for $\tilde{n} \geq 10$ as in Luo (2005a).

In Luo (2005a), the parameter $\alpha=-1$ is only considered as a blocking model and, as such, the model cannot be directly applied to the dynamics of $\mathrm{NAO}^{+}$ events $\left(m=2 \pi / L_{y}\right)$. Here, the theoretical model is naturally extended to include synoptic-scale eddies $(\alpha=1)$ that contribute toward the life cycle of the $\mathrm{NAO}^{+}$anomaly.

As noted in Luo (2005a), $J\left(\tilde{\psi}_{0}^{\prime}, \nabla^{2} \tilde{\psi}_{0}^{\prime}\right)_{P}$ plays a key role in the growth and decay of the preexisting planetary wave. The spatial structure of $J\left(\tilde{\psi}_{0}^{\prime}, \nabla^{2} \tilde{\psi}_{0}^{\prime}\right)_{P}$ determines the type of planetary waves that can grow and decay because it appears in the planetary-scale equation (2a) as a forcing term.

Substituting (4b) into $J\left(\tilde{\psi}_{0}^{\prime}, \nabla^{2} \tilde{\psi}_{0}^{\prime}\right)_{P}$ yields the preexisting eddy forcing (PEF):

$$
\begin{aligned}
F_{e} \approx & -\varepsilon^{3} J\left(\tilde{\psi}_{0}^{\prime}, \nabla^{2} \tilde{\psi}_{0}^{\prime}\right)_{P} \\
= & -i \alpha \frac{\left(\tilde{k}_{1}+\tilde{k}_{2}\right)^{2}\left(\tilde{k}_{2}-\tilde{k}_{1}\right) m}{4} \varepsilon^{3} f_{0}^{\prime 2} \exp \left\{i \left[\left(\tilde{k}_{2}-\tilde{k}_{1}\right) x\right.\right. \\
& \left.\left.-\left(\tilde{\omega}_{2}-\tilde{\omega}_{1}\right) t\right]\right\} \sin (m y)+\text { c.c. }
\end{aligned}
$$


It is evident that the preexisting eddy forcing has a meridional dipole structure due to the spatial structure of $\sin (m y)$ and can resonantly force the dipole NAO anomaly if the conditions $\left|k-\left(\tilde{k}_{2}-\tilde{k}_{1}\right)\right|=O(\varepsilon)$ and $\tilde{\omega}_{2}-\tilde{\omega}_{1}-\omega=O(\varepsilon)$ are satisfied. It can be confirmed that the two conditions are approximately satisfied in a moderate range of uniform background westerly wind, which allows a resonant interaction to occur between the dipole NAO anomaly and synoptic-scale eddies.

In the model, we fix the typical atmospheric parameters $L=10^{6} \mathrm{~m}, U=10 \mathrm{~m} \mathrm{~s}^{-1}, L_{y}=5(5000 \mathrm{~km}$ in dimensional form), and $F=1$ around the reference latitude $55^{\circ} \mathrm{N}$ when the dipole mode prior to the NAO is assumed to have a wavenumber-two structure $(n=$ 2). In addition, $\tilde{n}=10$ and $\Delta \tilde{n}=0.75$ are chosen such that (3b) is representative of synoptic-scale eddies as in Luo (2005a). The dependence of $\Delta \omega=\tilde{\omega}_{2}-\tilde{\omega}_{1}-\omega$ on the uniform background westerly wind $u_{0}$ is shown in Fig. 1. It is found that $\Delta \omega$ is small as the uniform background westerly wind is weak. This shows that the resonant interaction between the preexisting planetaryscale dipole mode and synoptic-scale eddies can take place in the presence of a weak background westerly wind. The zonally averaged wind in the troposphere over the North Atlantic is generally about $10 \mathrm{~m} \mathrm{~s}^{-1}$ $\left(u_{0}=1\right)$, and even less than $10 \mathrm{~m} \mathrm{~s}^{-1}$ in the higher latitudes (Franzke et al. 2004; Vallis et al. 2004). Thus, it is possible that there is a near-resonant interaction between the planetary-scale dipole mode and synopticscale eddies.

Following Luo (2005a), the second-order solution in (3a) and the forced nonlinear Schrodinger (NLS) equation describing the evolution of the dipole mode for $A$ can be obtained:

$$
\tilde{\psi}_{1}=-|A|^{2} \sum_{n=1}^{\infty} q_{n} g_{n} \cos (n+1 / 2) m y
$$

$$
\begin{aligned}
i\left(\frac{\partial A}{\partial T_{2}}+\right. & \left.C_{g} \frac{\partial A}{\partial X_{2}}\right)+\lambda \frac{\partial^{2} A}{\partial X_{1}^{2}}+\delta|A|^{2} A \\
& +G f_{0}^{\prime 2} \exp \left[-i\left(\Delta K X_{1}+\Omega T_{1}\right)\right]=0,
\end{aligned}
$$

where

$$
G=\alpha \sqrt{\frac{L_{y}}{2}} \frac{\left(\tilde{k}_{1}+\tilde{k}_{2}\right)^{2}\left(\tilde{k}_{2}-\tilde{k}_{1}\right) m}{4\left(k^{2}+m^{2}+F\right)},
$$

$k-\left(\tilde{k}_{2}-\tilde{k}_{1}\right)=\Delta k=\varepsilon \Delta K, \tilde{\omega}_{2}-\tilde{\omega}_{1}-\omega=\Delta \omega=\varepsilon \Omega$, $q_{n}, g_{n}$, and the other coefficients can be found in Luo (2005a).

Note that solution (6a), which is independent of fast variables $x$ and $t$, is a modification of uniform background flow $u_{0}$ due to the nonlinear self-interaction

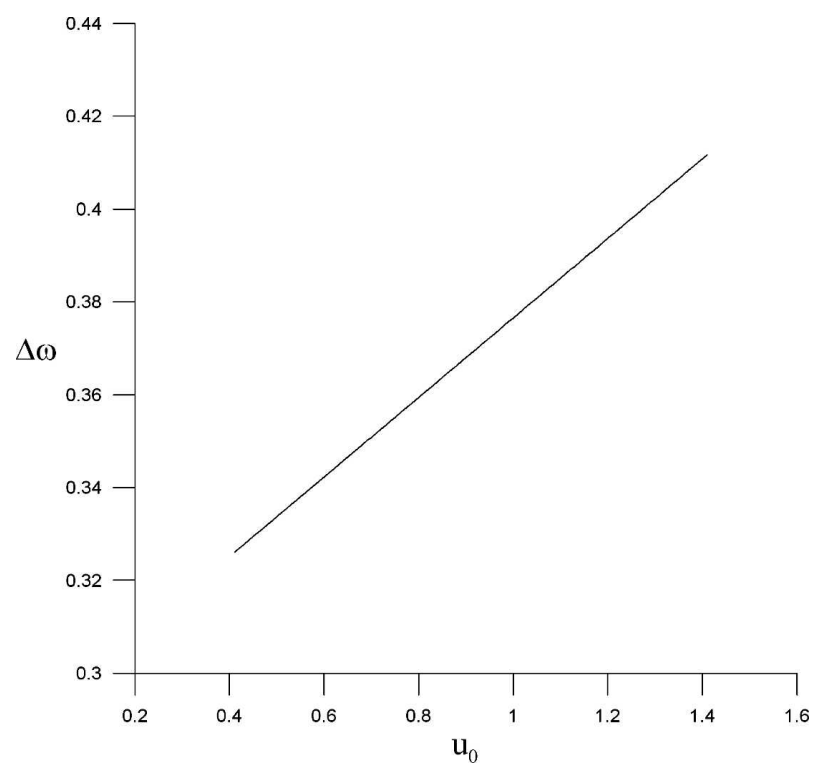

FIG. 1. Dependence of $\Delta \omega=\tilde{\omega}_{2}-\tilde{\omega}_{1}-\omega$ with the uniform basic westerly wind $u_{0}$.

(vorticity flux) of the eddy-driven dipole mode. This actually represents a feedback by the dipole NAO anomaly onto the zonal mean flow if the dipole mode is amplified into a NAO pattern. Thus (6) can tell us how the NAO circulation is driven by synoptic-scale eddies and how the zonal mean flow responds to the NAO. In previous theoretical NAO models, these analytical solutions cannot be found. In section 6, (6a) will be used to discuss the dynamical relationship between the NAO and zonal index. In addition, it is inferred that the zonal mean flow anomalies are indirectly driven by synopticscale eddies through the self-interaction of the excited NAO anomaly in that the NAO dipole anomaly is directly driven by the synoptic- scale eddies. In the $\mathrm{NH}$, climatological stationary waves forced by land-sea contrast topography and heating play a certain role in a change in a zonal mean flow (DeWeaver and Nigam 2000), but the issue is beyond the scope of this work.

It is found from (6b) that the evolution equation of the dipole NAO anomaly associated with synoptic-scale eddies is similar in form to the equation of an eddyinduced dipole block derived by Luo (2005a). But the present model can be used to investigate how synopticscale eddies reinforce dipole modes for different phases because of the inclusion of $\alpha=\mp 1$ in (6b). Equation (6b) represents the evolution of an $\mathrm{NAO}^{+}$event if $m=$ $2 \pi / L_{y}$ is allowed in (4a). In contrast, it denotes the evolution of an $\mathrm{NAO}^{-}$event if $m=-2 \pi / L_{y}$ is chosen. However, whether an $\mathrm{NAO}^{+}\left(\mathrm{NAO}^{-}\right)$dipole anomaly forms is dependent upon the spatial structure of the PEF in (5). 
Figure 2 shows the dependence of the period $T_{R}=$ $2 \pi / \Delta \omega$ in the PEF on the uniform westerly wind $\left(u_{0}\right)$. It is found that the period $T_{R}$ of the PEF decreases as the background westerly wind $u_{0}$ increases. The period corresponds to about 18 to 22 days as $u_{0}$ ranges from 0.4 to 1.4 (from 4 to $14 \mathrm{~m} \mathrm{~s}^{-1}$ in the dimensional form). It is anticipated that the dipole NAO anomaly should possess such a period. However, the actual life cycle period of observed NAO events may be affected by other factors such as the zonal mean flow advection and dissipation and so on.

\section{c. Analytical solution of the feedback of dipole $N A O$ anomaly on preexisting synoptic-scale waves}

As pointed out by Benedict et al. (2004), for the $\mathrm{NAO}^{+}$and $\mathrm{NAO}^{-}$events the onset of the NAO is attributable to synoptic-scale waves, but the synopticscale waves undergo a deformation (amplifying, decaying, merging and breaking) when entering the NAO region. To represent the interaction between the NAO events and synoptic-scale waves, it is useful to obtain the second-order solution for $\tilde{\psi}_{1}^{\prime}$ in (3b). The secondorder solution of synoptic-scale waves due to the feedback of the dipole NAO anomaly can be obtained by substituting (4) into the second-order equation of synoptic-scale waves derived from (2b) (Luo 2005a):

$$
\begin{aligned}
\tilde{\psi}_{1}^{\prime}= & -\frac{m}{4} \sqrt{\frac{2}{L_{y}}} Q_{1} A f_{0}^{\prime} \exp \left\{i \left[\left(\tilde{k}_{1}+k\right) x\right.\right. \\
& \left.\left.-\left(\tilde{\omega}_{1}+\omega\right) t\right]\right\}\left[p_{1} \sin \left(\frac{3 m}{2} y\right)+r_{1} \sin \left(\frac{m}{2} y\right)\right] \\
& -\alpha \frac{m}{4} \sqrt{\frac{2}{L_{y}}} Q_{2} A f_{0}^{\prime} \exp \left\{i \left[\left(\tilde{k}_{2}+k\right) x\right.\right. \\
& \left.\left.-\left(\tilde{\omega}_{2}+\omega\right) t\right]\right\}\left[p_{2} \sin \left(\frac{3 m}{2} y\right)+r_{2} \sin \left(\frac{m}{2} y\right)\right] \\
& +\frac{m}{4} \sqrt{\frac{2}{L_{y}}} A^{*} Q_{1} f_{0}^{\prime} \exp \left\{i \left[\left(\tilde{k}_{1}-k\right) x\right.\right. \\
& \left.\left.-\left(\tilde{\omega}_{1}-\omega\right) t\right]\right\}\left[s_{1} \sin \left(\frac{3 m}{2} y\right)+h_{1} \sin \left(\frac{m}{2} y\right)\right] \\
& +\alpha \frac{m}{4} \sqrt{\frac{2}{L_{y}}} A^{*} Q_{2} f_{0}^{\prime} \exp \left\{i \left[\left(\tilde{k}_{2}-k\right) x\right.\right. \\
& \left.\left.-\left(\tilde{\omega}_{2}-\omega\right) t\right]\right\}\left[s_{2} \sin \left(\frac{3 m}{2} y\right)+h_{2} \sin \left(\frac{m}{2} y\right)\right] \\
& + \text { c.c. },
\end{aligned}
$$

where $Q_{j}=k^{2}+m^{2}-\left(\tilde{k}_{j}^{2}+m^{2} / 4\right)(j=1,2)$ and the other coefficients in (7) are given in the appendix.

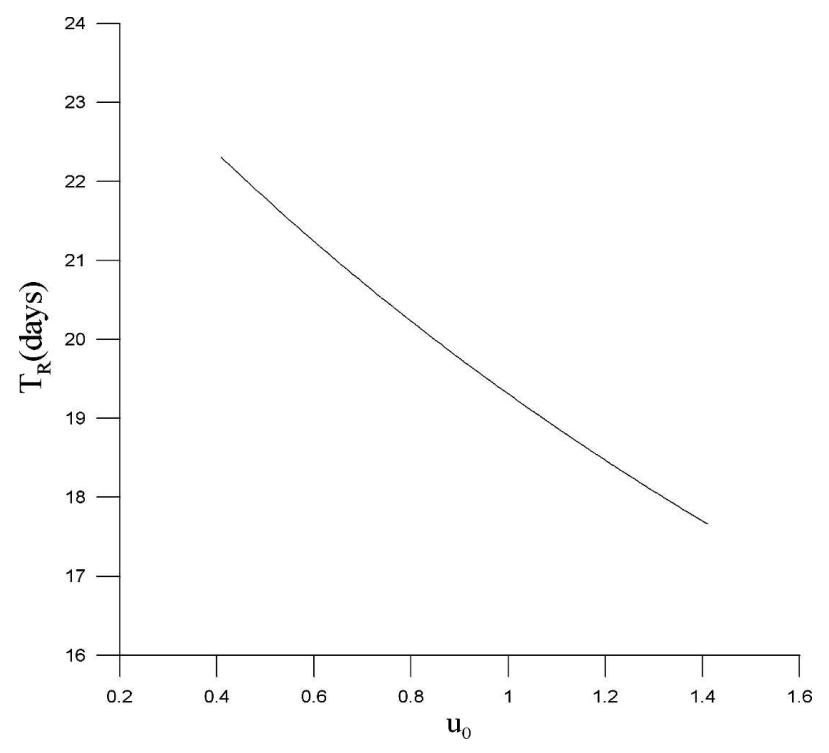

FIG. 2. Period $T_{R}=2 \pi / \Delta \omega$ of preexisting eddy forcing in the different background westerly wind $u_{0}$.

Note that, when $m=-2 \pi / L_{y}$ and $\alpha=-1$ are chosen, (7) reduces to the solution for the deformation of synoptic-scale eddies by the feedback of blocked flow obtained by Luo (2005a). Thus, solution (7) predicts a change in the synoptic-scale waves induced by the evolution of a NAO event. Using solution (7) and the solution to (6b) can give some insight into how the dipole NAO mode and synoptic-scale waves deform during their interaction.

To obtain the solutions of the interaction between planetary-scale dipole mode and synoptic-scale eddies leading to the NAO, it is convenient for us to change (6b) into a fast-variable equation in $t-x$ space. If we define $B(x, t)=\varepsilon A\left(\varepsilon x, \varepsilon^{2} x, \varepsilon t, \varepsilon^{2} t\right), f_{0}(x)=\varepsilon^{3 / 2} f_{0}^{\prime}(\varepsilon x)=$ $a_{0} \exp \left[-\mu \varepsilon^{2}\left(x+x_{0}\right)^{2}\right]$, and $a_{0}=\varepsilon^{3 / 2} a_{0}^{\prime}$, then the forced NLS equation for (6b) can be rewritten as

$$
\begin{aligned}
i\left(\frac{\partial B}{\partial t}+C_{g}\right. & \left.\frac{\partial B}{\partial x}\right)+\lambda \frac{\partial^{2} B}{\partial x^{2}}+\delta|B|^{2} B \\
& +G f_{0}^{2} \exp [-i(\Delta k x+\Delta \omega t)]=0 .
\end{aligned}
$$

In this case, the solutions for planetary-scale waves and synoptic-scale eddies in the fast-variable form are expressed as

$$
\begin{aligned}
\psi_{P} \approx & -u_{0} y+\varepsilon \tilde{\psi}_{0}+\varepsilon^{2} \tilde{\psi}_{1}=-u_{0} y \\
& +B \sqrt{\frac{2}{L_{y}}} \exp [i(k x-\omega t)] \sin (m y)+\text { c.c. }+\psi_{a},
\end{aligned}
$$




$$
\begin{aligned}
\psi_{a}= & \varepsilon^{2} \tilde{\psi}_{1}=-|B|^{2} \sum_{n=1}^{\infty} q_{n} g_{n} \cos (n+1 / 2) m y, \\
\psi^{\prime} \approx & \varepsilon^{3 / 2}\left(\tilde{\psi}_{0}^{\prime}+\varepsilon \tilde{\psi}_{1}^{\prime}\right)=\psi_{0}^{\prime}+\psi_{1}^{\prime}, \\
\psi_{0}^{\prime}= & f_{0}(x)\left\{\exp \left[i\left(\tilde{k}_{1} x-\tilde{\omega}_{1} t\right)\right]\right. \\
& \left.+\alpha \exp \left[i\left(\tilde{k}_{2} x-\tilde{\omega}_{2} t\right)\right]\right\} \sin \left(\frac{m}{2} y\right)+\text { c.c. }, \quad(9 \mathrm{~d}) \\
\psi_{1}^{\prime}= & -\frac{m}{4} \sqrt{\frac{2}{L_{y}}} Q_{1} B f_{0} \exp \left\{i \left[\left(\tilde{k}_{1}+k\right) x\right.\right. \\
& \left.\left.-\left(\tilde{\omega}_{1}+\omega\right) t\right]\right\}\left[p_{1} \sin \left(\frac{3 m}{2} y\right)+r_{1} \sin \left(\frac{m}{2} y\right)\right] \\
& -\alpha \frac{m}{4} \sqrt{\frac{2}{L_{y}}} Q_{2} B f_{0} \exp \left\{i \left[\left(\tilde{k}_{2}+k\right) x\right.\right. \\
& \left.\left.-\left(\tilde{\omega}_{2}+\omega\right) t\right]\right\}\left[p_{2} \sin \left(\frac{3 m}{2} y\right)+r_{2} \sin \left(\frac{m}{2} y\right)\right] \\
& +\frac{m}{4} \sqrt{\frac{2}{L_{y}}} Q_{1} B^{*} f_{0} \exp \left\{i \left[\left(\tilde{k}_{1}-k\right) x\right.\right. \\
& \left.\left.-\left(\tilde{\omega}_{1}-\omega\right) t\right]\right\}\left[s_{1} \sin \left(\frac{3 m}{2} y\right)+h_{1} \sin \left(\frac{m}{2} y\right)\right] \\
& +\alpha \frac{m}{4} \sqrt{\frac{2}{L_{y}}} Q_{2} B^{*} f_{0} \exp \left\{i \left[\left(\tilde{k}_{2}-k\right) x\right.\right. \\
& \left.\left.-\left(\tilde{\omega}_{2}-\omega\right) t\right]\right\}\left[s_{2} \sin \left(\frac{3 m}{2} y\right)+h_{2} \sin \left(\frac{m}{2} y\right)\right] \\
& + \text { c.c. }
\end{aligned}
$$
condition Fig. 3 shows the planetary-scale field $\left(\psi_{P}\right)$ synoptic-scale field $\left(\psi^{\prime}\right)$, and total field $\left(\psi_{T}=\psi_{P}+\psi^{\prime}\right)$ during the interaction between an incipient negativephase NAO and preexisting synoptic-scale waves upstream of the region where the low-frequency NAO anomaly develops for the parameters $u_{0}=0.7, \mu=1.2$, $a_{0}=0.17, \varepsilon=0.24, x_{0}=2.87 / 2, m=-2 \pi / L_{y}$, and $\alpha=$ -1 for which the incipient NAO is stationary and synoptic-scale eddies are located upstream of the NAO region.

In Fig. 3a, a typical $\mathrm{NAO}^{-}$(high over low) event can form from an initially weak high-over-low dipole due to the positive feedback of the PEF (day 9). After day 10, the NAO will decay due to its negative feedback until day 21. Afterwards, the second life cycle of this NAO anomaly reappears owing to the repeated forcing of upstream synoptic-scale eddies. However, because the diffluent flow at day 21, considered as an incipient NAO anomaly of the second NAO event, is weaker than that at day 0 , the second NAO event is inevitably weaker than the first NAO event. But the basic characteristics of the first and following NAO events are similar and consistent with those of an observed $\mathrm{NAO}^{-}$ event (see Fig. 8 of Benedict et al. 2004). Thus, the $\mathrm{NAO}^{-}$event can be driven by preexisting synoptic eddies upstream of the NAO region, implicating that the NAO is a nonlinear initial-value problem (Franzke et al. 2004). During the life cycle of the NAO event the positive and negative feedbacks of the PEF from synoptic-scale eddies seem to determine the growth and decay of the NAO event. This result is new and different from the diagnostic result of the NAO by Feldstein (2003), who finds that the synoptic-scale eddies maintain the NAO anomaly, but the low-frequency eddies contribute toward the decay of the NAO. In our model, on one hand, the PEF can make the NAO grow, but then, on the other hand, make the NAO decay, reflecting the duality of the PEF. In fact, the negative role of the PEF is similar to the role of the Ekman pumping of low-frequency eddies in causing the NAO decay noted by Feldstein (2003) since $-\boldsymbol{\nabla} \cdot \overline{\left(\mathbf{V}^{\prime} q^{\prime}\right)}$ can be parameterized as $-\gamma_{e} \nabla^{2} \psi\left(\gamma_{E}\right.$ is the dissipation coefficient) in a large-scale form. Such a synoptic-scale eddy param- 
(a)

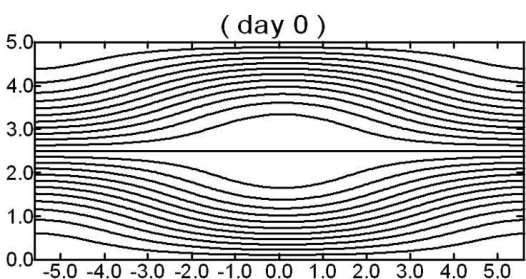

(day 6 )

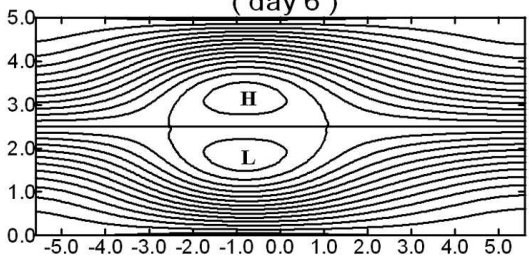

(day 12 )

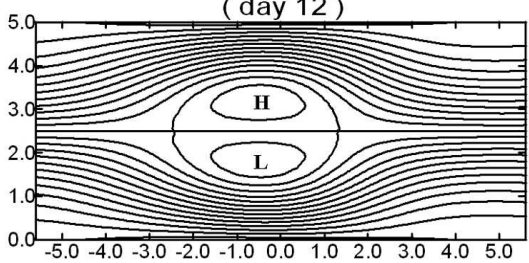

(day 18 )

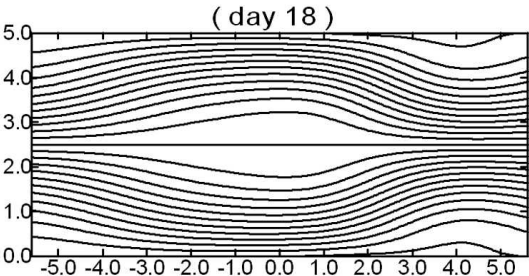

(day 24 )

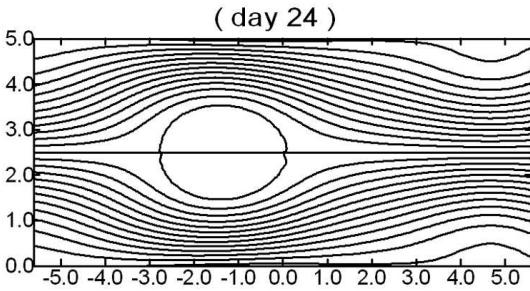

(day 30 )

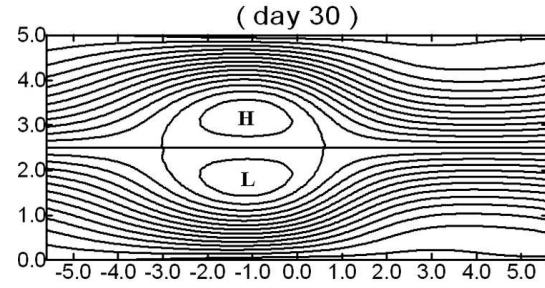

(day 36 )

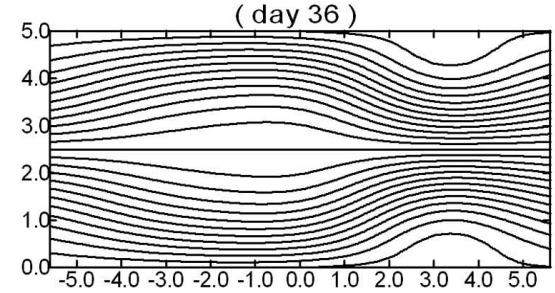

(day 3 )

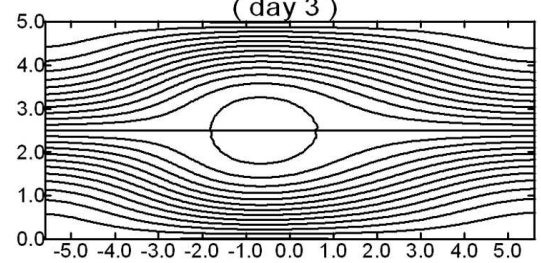

(day 9 )

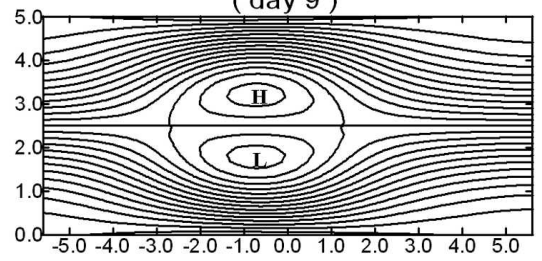

(day 15 )

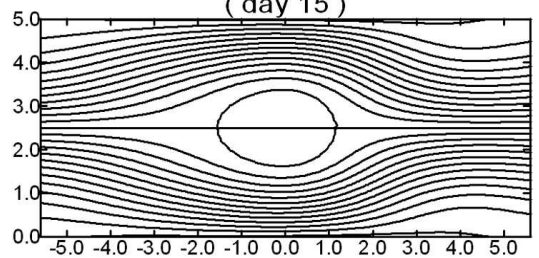

(day 21)

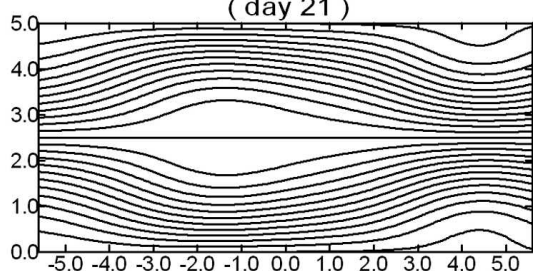

(day 27 )

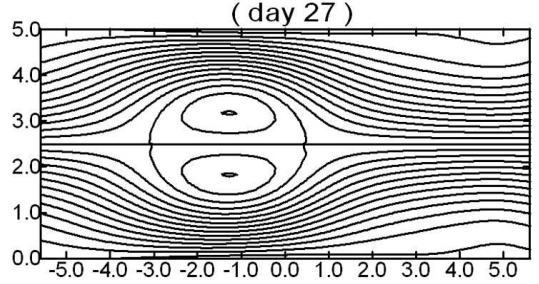

(day 33 )

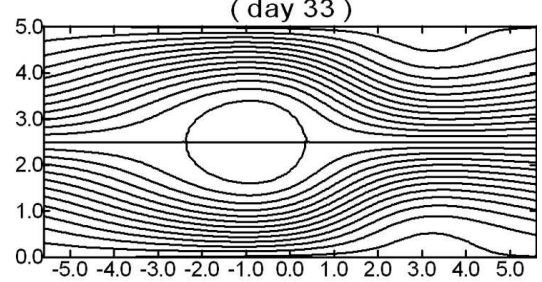

(day 39 )

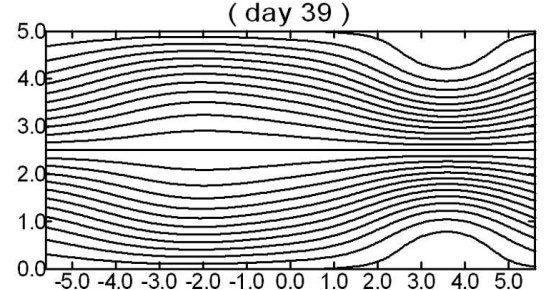

FIG. 3. (See facing page for caption) 
(b)
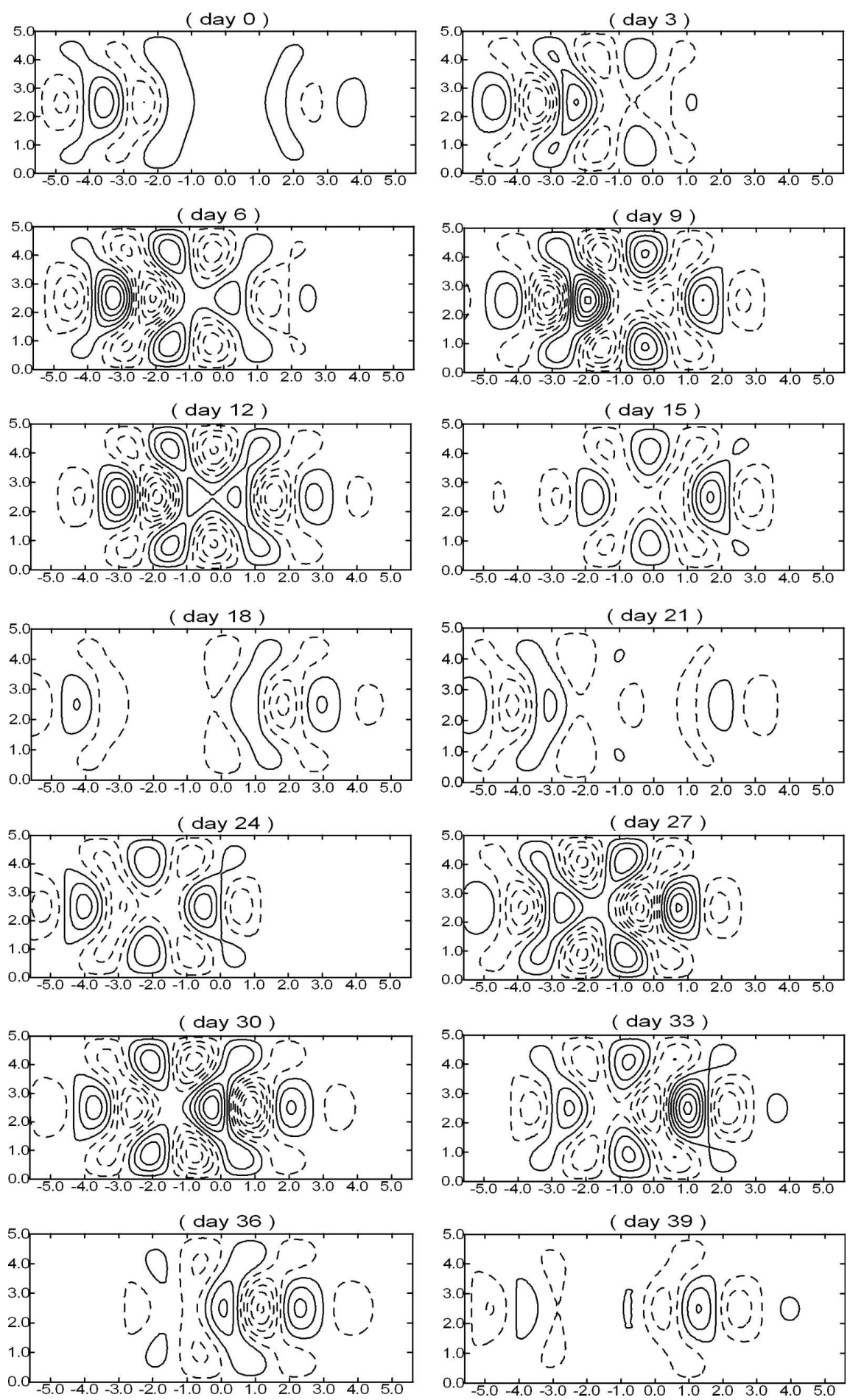

FIG. 3. Interaction between an incipient high-over-low structure (preexisting negative-phase $\mathrm{NAO}$ anomaly) and preexisting synoptic-scale waves for the parameters $u_{0}=0.7, \mu=1.2$, $a_{0}=0.17, \varepsilon=0.24, x_{0}=2.87 / 2, m=-2 \pi / L_{y}$, and $\alpha=-1$, and for the initial value of $B(x, 0)$ $=B_{0}$ and $B_{0}=0.35$ : (a) planetary-scale field $\left(\psi_{P}\right)$, in which the contour interval $(\mathrm{CI})$ is 0.15 , (b) synoptic-scale field $\left(\psi^{\prime}\right)(\mathrm{CI}=0.3)$, in which the dashed curve denotes the negative streamfunction and the solid curve denotes the positive streamfunction, and (c) total field $\left(\psi_{T}=\psi_{P}+\psi^{\prime}\right)(\mathrm{CI}=0.3)$. 
(c)
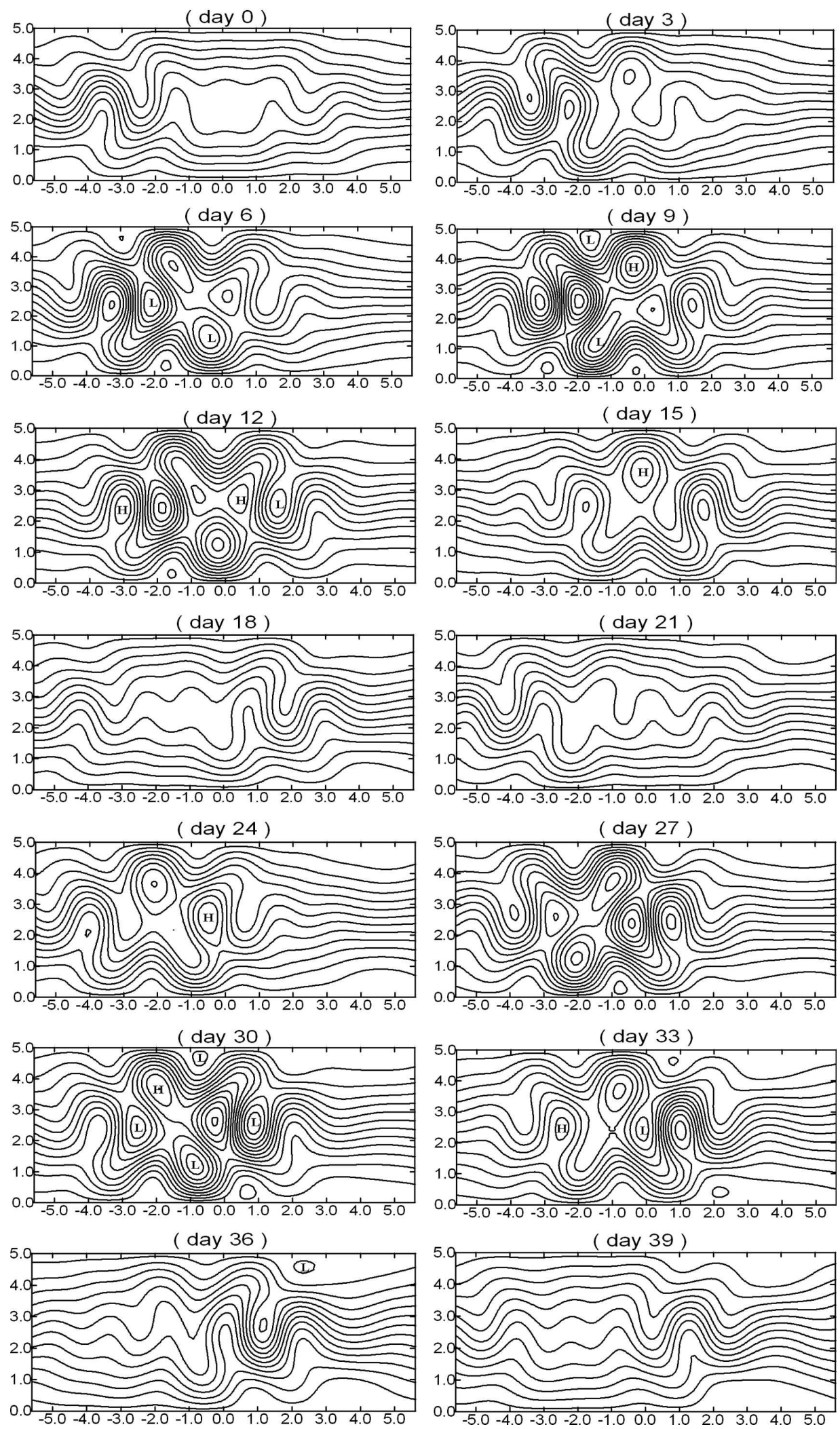

FIG. 3. (Continued) 
eterization fails to reflect the positive feedback of synoptic-scale eddies that maintain the NAO anomalies. This shortcoming can be more or less avoided in our weakly nonlinear NAO model. As we will find in the next subsection, the positive and negative feedbacks of the PEF is a natural feature of synoptic-scale eddies that drive the NAO life cycle.

Figure $3 \mathrm{~b}$ shows that the synoptic-scale waves tend to enhance and split into two branches, consistent with the behavior of the synoptic waves during a dipole block episode found numerically by Shutts (1983) and observed by Nakamura and Wallace (1993).

Figure $3 \mathrm{c}$ shows the total field of the corresponding $\mathrm{NAO}^{-}$event. The time sequences of the total field exhibit a close resemblance to the life cycle of an observed $\mathrm{NAO}^{-}$event presented by Benedict et al. (2004, see their Fig. 4), which looks similar to observed meandering blocking flows (Berggren et al. 1949; Lupo and Smith 1995a,b). Inspecting Fig. 3c demonstrates that when the synoptic-scale troughs and ridges, existing upstream of the NAO region, propagate into the NAO region, they amplify, merge, and then break. In this process, the warm air moves into the northern part of the NAO region with the amplifying ridge, but the cold air drifts into the southern part of the NAO region with deepening troughs. This theoretical result looks similar to observed $\mathrm{NAO}^{-}$events presented by Benedict et al. (2004, their Figs. 4 and 6).

It should be pointed out that the magnitude and distribution of $f_{0}^{\prime}$ also affect the excitement of the NAO event. For example, when $f_{0}^{\prime}$ is of a large amplitude, a stronger NAO event can form (not shown). However, only a weak NAO event is formed when the synoptic waves are to the east of the incipient NAO anomaly and far from the NAO region (not shown). But when the synoptic waves are in a moderate position upstream of the incipient NAO anomaly, a strong NAO event can be excited (not shown). It can also be noted that varying $G, \Delta K$, and $\Omega$ does not strongly affect the basic physics of eddy-driven NAO events when $\tilde{k}_{2}-\tilde{k}_{1}$ is close to $k$ (zonal wavenumber of NAO) (not shown).

It must be noted that because $\psi_{1}^{\prime}$ includes the amplitude $A$ of the dipole mode, it becomes larger so that $\psi_{1}^{\prime}$ is the same as or exceeds $\psi_{0}^{\prime}$ when the dipole NAO mode grows and is very strong. In this case, asymptotic theory is violated because the strongly nonlinear process is dominant. This is the difficult problem encountered in weakly nonlinear theory (Malguzzi and Malanotte-Rizzoli 1984; Luo 2005a). Even so, the weakly nonlinear analytical solutions here can tell us more information about what processes drive the NAO and what factors determine the phase of the NAO.

Generally speaking, the lifetime of the NAO events is dominated by the period of the PEF, but is also dependent upon the strength of the planetary-scale dipole mode prior to the NAO, the setting of the background westerly wind, and frictional effects. If the amplitude of the preexisting planetary wave is stronger, the eddyexcited dipole mode can easily reach the amplitude required by the defined NAO event. In this case, the life period of the NAO events should be longer (not shown). Although the period of the PEF is about 21 days for our parameters, the lifetime of observed NAO events does not strictly correspond to this time scale because the NAO anomaly is relatively weak at days 18 and 21 (Figs. 3a and 3c). But, crudely speaking, the time scale of the NAO life cycle is approximately two weeks. In an idealized model, Vallis et al. (2004) noted that the higher-frequency eddies can produce the NAO with time scales of 10 to 50 days in a certain range of frictional effects. A comparison with the synoptic study of NAO events by Benedict et al. (2004) indicates that the theoretical model here can capture the physical essence of the interaction between synoptic-scale eddies and low-frequency dipole modes leading to an $\mathrm{NAO}^{-}$ event.

\section{b. Physical mechanism of negative-phase NAO events}

For the same parameters as in Fig. 3, the time evolution of the PEF for $F_{e} \approx-\varepsilon^{3} J\left(\tilde{\psi}_{0}^{\prime}, \nabla^{2} \tilde{\psi}_{0}^{\prime}\right)_{P}$ is shown in Fig. 4, which corresponds to the forcing of eddy vorticity.

In Fig. 4, we note that, at day $0, F_{e}$ possesses an isolated negative-over-positive dipole structure, which corresponds to an isolated anticyclonic-over-cyclonic vorticity forcing pattern, and exists upstream of an incipient dipole NAO. Since $F_{e}$ is time-dependent, the time evolution of $F_{e}$ is actually an indicator of whether synoptic-scale waves drive the $\mathrm{NAO}^{-}$event to grow or decay. Before day $9, F_{e}$ has mainly a negative-overpositive structure upstream of the NAO region, but a reverse structure appears upstream after day 9. Thus, before day 9 , the synoptic-scale waves will repeatedly transport anticyclonic vorticities into the northern part of the downstream NAO anomaly and cyclonic vorticities into the southern part, indicating that the PEF plays a positive feedback role. This process will make the $\mathrm{NAO}^{-}$anomaly grow and resembles the eddystretching mechanism of Shutts $(1983,1986)$ for blocking events. After day 9 there exists a reverse trend because $F_{e}$ exhibits a spatial pattern opposite to that before day 9 , which makes the NAO anomaly decay. For this case, the PEF plays a negative feedback role, which is similar to the role played by the Ekman pumping in the NAO decay (Feldstein 2003). But after day 21, a 

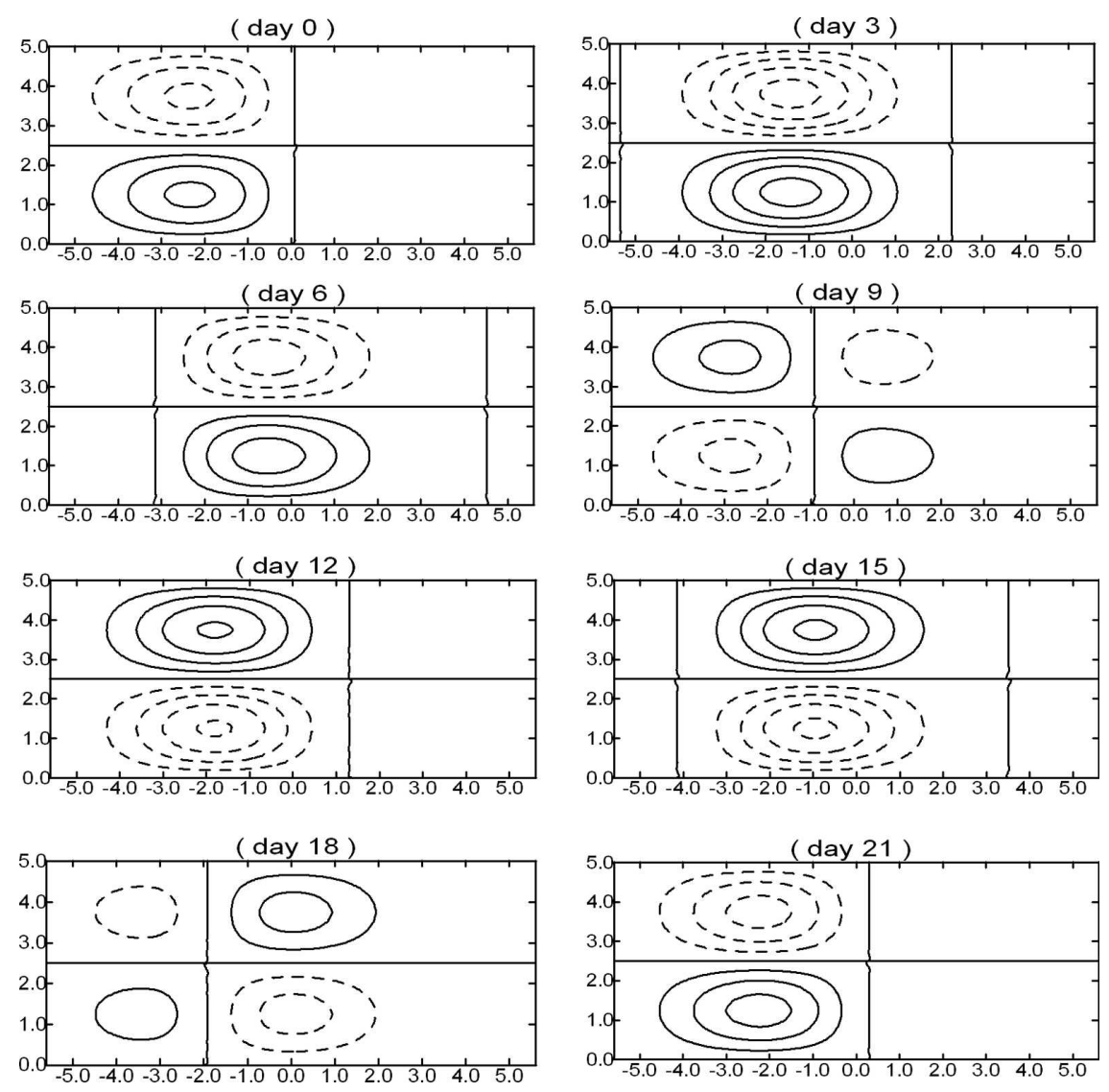

FIG. 4. Evolution of eddy forcing $F_{e}$ from preexisting synoptic-scale waves that allow the negative NAO phase to occur for the same parameters in Fig. $3(\mathrm{CI}=0.05)$.

spatial pattern of $F_{e}$ similar to that before day 9 reappears upstream of the NAO region, and will lead to the recurrence of an $\mathrm{NAO}^{-}$event. Thus, whether an $\mathrm{NAO}^{-}$event forms and persists depends essentially upon if an incipient dipole (weak high-over-low) NAO $\left(m=-2 \pi / L_{y}\right)$ matches the spatial structure of the PEF $\left(F_{e}\right)$ for $\alpha=-1$.

We have demonstrated here that the preexisting synoptic-scale waves must be required to make the PEF $\left(F_{e}\right)$ have the same spatial structure as in Fig. 4 in order to allow a two-week $\mathrm{NAO}^{-}$event to form. Of course, the incipient NAO anomaly must have a high-over-low dipole structure. As found in Luo et al. (2001), the observed eddy forcing prior to the block onset has in general the same spatial structure as in Fig. 4 at day 0 in order to allow a block circulation to be excited. Thus, it is concluded that the planetary-scale high-over-low flow and associated synoptic-scale waves having the eddy vorticity forcing in Fig. 4 prior to the NAO onset are likely to be a precursor of the $\mathrm{NAO}^{-}$life cycle. The periodic variation of the spatial structure of the PEF in Fig. 4 reflects that the life cycle of the NAO event is a manifestation of natural variability in eddy-driven lowfrequency dipole mode.

\section{Onset of eddy-driven positive-phase NAO events and associated physical mechanism}

\section{a. Life cycle of positive-phase NAO events and onset of downstream blocks}

Given the initial values for $B(x, 0)=B_{0} \cos (\varepsilon x)$ with $B_{0}=0.45$, the instantaneous planetary-scale field, synoptic-scale field, and the total field of the interaction between an incipient $\mathrm{NAO}^{+}$dipole anomaly $(m=2 \pi /$ $\left.L_{y}\right)$ and preexisting synoptic-scale waves $(\alpha=1)$ are shown in Fig. 5 for the same parameters as in Fig. 3.

In Fig. 5a, the incipient $\mathrm{NAO}^{+}$has a low-over-high structure prior to NAO onset, which is a planetaryscale precursor for the onset of an eddy-driven $\mathrm{NAO}^{+}$ event different from those required for the onset of $\mathrm{NAO}^{-}$events (e.g., $m=-2 \pi / L_{y}$ and $\alpha=-1$ ).

The $\mathrm{NAO}^{+}$pattern shown in Fig. 5a resembles the composite field of observed $\mathrm{NAO}^{+}$events presented by Benedict et al. (2004, their Fig. 3) and exhibits a period 
of about two weeks, consistent with the time scale of observed NAO events (Feldstein 2003). The NAO anomaly completes a life cycle from day 0 to 15 and seems to reach its strongest state at day 9. At the same time, a weak high-over-low dipole structure appears downstream of the decayed $\mathrm{NAO}^{+}$region at day 15 . This weak dipole structure can be amplified into a strong dipole block through the continued forcing of upstream synoptic-scale waves. The region where the strong dipole block occurs corresponds approximately to the Europe sector if the low-over-high pattern before day 15 is assumed to occur in the Atlantic sector. This typical dipole block can recur over the Europe sector. This shows that the breakdown of $\mathrm{NAO}^{+}$events favors the occurrence of dipole blocks in the Europe sector. Thus, it can be concluded that the blocking frequency in the European sector should be higher during an $\mathrm{NAO}^{+}$year than during an $\mathrm{NAO}^{-}$year.

In previous studies, the NAO index is usually defined as the difference between the normalized mean winter sea level pressure (SLP) anomalies in the Azores and Iceland. The phase of the NAO is reflected by the average value of the NAO index from December to February or March. When the seasonal mean NAO index is negative (positive), it represents the $\mathrm{NAO}^{-}\left(\mathrm{NAO}^{+}\right)$ phase. This does not imply that $\mathrm{NAO}^{-}$events cannot occur during the positive phase of the seasonal mean $\mathrm{NAO}$ index. In fact, if $\mathrm{NAO}^{-}$events with life times of nearly two weeks occur more frequently than the positive-phase events in the winter season, the seasonal mean NAO index should be negative in this season unless the $\mathrm{NAO}^{+}$events are strong enough, as implied by the recent synoptic study of the NAO by Benedict et al. (2004).

Shabbar et al. (2001) found that blocking events are likely to occur during the $\mathrm{NAO}^{-}$phase. Seemingly, then, $\mathrm{NAO}^{-}$events do not correspond to blocking events. In fact, however, the $\mathrm{NAO}^{-}$events are essentially identical to blocking events over the North Atlantic because the $\mathrm{NAO}^{-}$events exhibit strong similarity to blocking events in many aspects (lifetime, pattern, and dynamical origin). This point can be seen in the daily and composite fields of $\mathrm{NAO}^{-}$events (Benedict et al., 2004, their Figs. 4 and 5), which is also verified in the unfiltered fields of $\mathrm{NAO}^{-}$events presented in the next section. If blocking events occur frequently over the North Atlantic, the seasonal mean NAO index is negative. In this case, blocking events over the North Atlantic are most likely to occur during the negative phase of the NAO (a negative value of the seasonal mean NAO index) (Shabbar et al. 2001). However, if $\mathrm{NAO}^{+}$events occur frequently or blocking events are infrequent, the seasonal mean NAO index is positive.
Thus, blocking events are unlikely to occur during the positive phase of the NAO. Even so, the assertion that the $\mathrm{NAO}^{-}$events are identical to blocking events over the North Atlantic does not contradict the observational study of Shabbar et al. because the seasonal mean value of the NAO index is used to characterize the phase of the NAO in their study.

Although Shabbar et al. (2001) found a very weak correlation between NAO and blocking activity over Europe, they noted that when the NAO transits from the predominantly negative phase (1960-70) into a mainly positive phase (1980-90) there exists a marked increase in the block frequency over Europe. Thus, the marked increase of the European blocking frequency during the positive NAO phase may be due to the influence of upstream $\mathrm{NAO}^{+}$events. Observational studies by Quadrelli et al. (2001) and Scherrer et al. (2006) shows a high (statistically significant) correlation between the positive NAO phase and Atlantic region blocking and positive NAO phase and European blocking. A similar observational study of Barriopedro et al. (2006) also confirms this result. Next, we will present observational evidence to support this theoretical result.

On the other hand, we find in Fig. 5b that the deformation of synoptic-scale waves during the life cycle of a $\mathrm{NAO}^{+}$event differs significantly from that of a $\mathrm{NAO}^{-}$ episode. For the $\mathrm{NAO}^{+}$event the synoptic-scale waves that propagate toward the east almost along a straight line weaken during the intensification stage of a lowover-high flow pattern and intensify during the decay stage, respectively. Such behavior of the synoptic-scale eddies associated with the onset of the $\mathrm{NAO}^{+}$event is remarkably different from the evolution of synoptic eddies during the $\mathrm{NAO}^{-}$episode.

Figure $5 \mathrm{c}$ clearly shows that initially strong synopticscale ridges and troughs appear upstream of the NAO region. They tend to decay during their interaction with an incipient $\mathrm{NAO}^{+}$anomaly. At day 9, these synoptic ridges and troughs almost disappear and a strong zonal westerly jet prevails over the Atlantic sector. Then both strong synoptic-scale ridges and troughs reappear upstream of the NAO region and a high-over-low structure occurs downstream. Thus, in the growth stage of the $\mathrm{NAO}^{+}$event, the synoptic-scale waves appear to be absorbed by the zonal westerly jet and eventually form a jet intensification anomaly (cold-over-warm or lowover-high), but there exists a reverse trend during the decay stage of the $\mathrm{NAO}^{+}$event. This process can be more clearly seen in the daily fields of the $\mathrm{NAO}^{+}$ events presented by Benedict et al. (2004, their Fig. 2). After the $\mathrm{NAO}^{+}$event has decayed, the synoptic- 
(a)
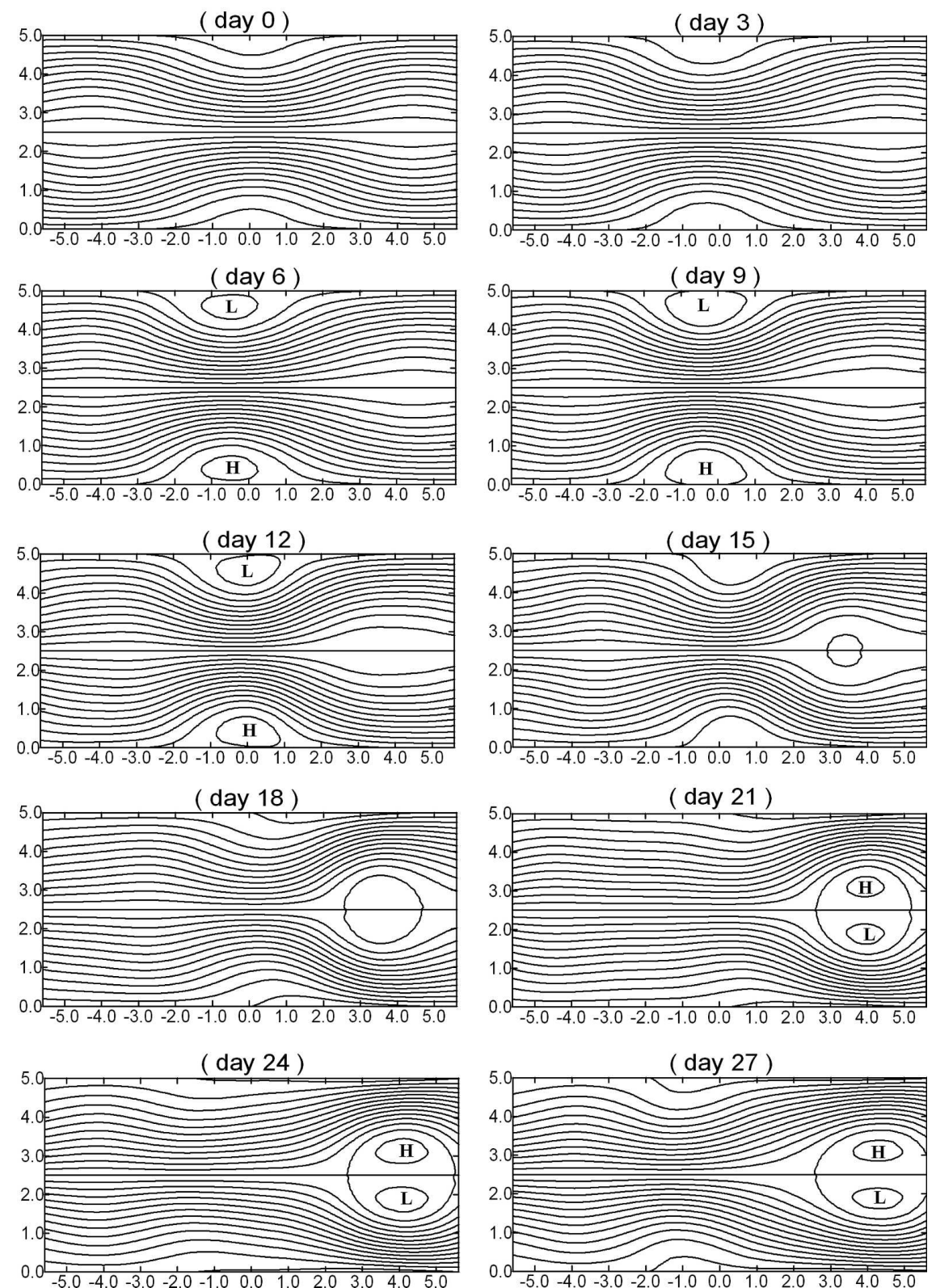

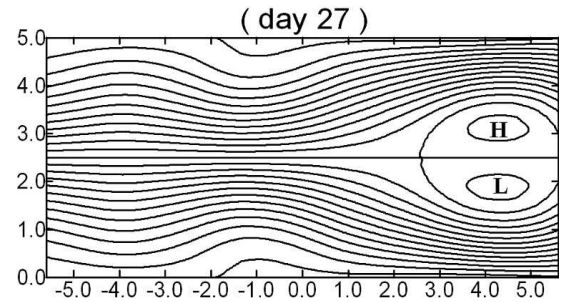

FIG. 5. Interaction between an incipient low-over-high structure (preexisting positive-phase NAO anomaly for $m=2 \pi / L_{y}$ and $\alpha=1$ ) and preexisting synoptic-scale waves for the parameters same as in Fig. 3 but for the initial value of $B(x, 0)=B_{0} \cos (\varepsilon x)$ and $B_{0}=0.45$ : (a) planetary-scale field $\left(\psi_{P}\right)$, in which the contour interval (CI) is 0.15 , (b) synoptic-scale field $\left(\psi^{\prime}\right)(\mathrm{CI}=0.3)$, and $(\mathrm{c})$ total field $\left(\psi_{T}=\psi_{P}+\psi^{\prime} ; \mathrm{CI}=0.3\right)$.

scale ridges and troughs again amplify and merge across the North Atlantic into Europe and eventually form a large-scale high-over-low structure similar to a dipole block often observed in the Europe sector. Even in the total field, the dipole pattern of a block is also particularly evident during the period from day 21 to 27 and from day 36 to 45 . Thus, the breakdown of the $\mathrm{NAO}^{+}$ event can accompany the subsequent establishment of a long-lived downstream dipole block, especially over the Europe sector. This will be shown using observational cases in the next section. For the initial value of $B(x, 0)=B_{0}$ with $B_{0}=0.45$, a similar result is found, but the life period of the European block is rather long (not shown). 


\section{(a) continued}
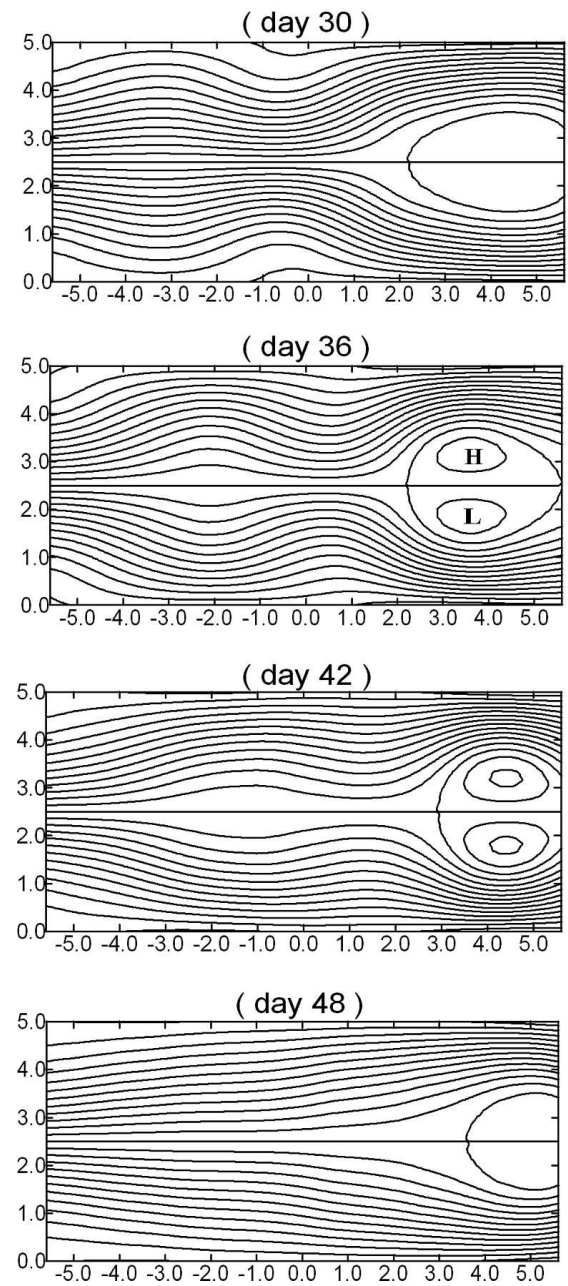
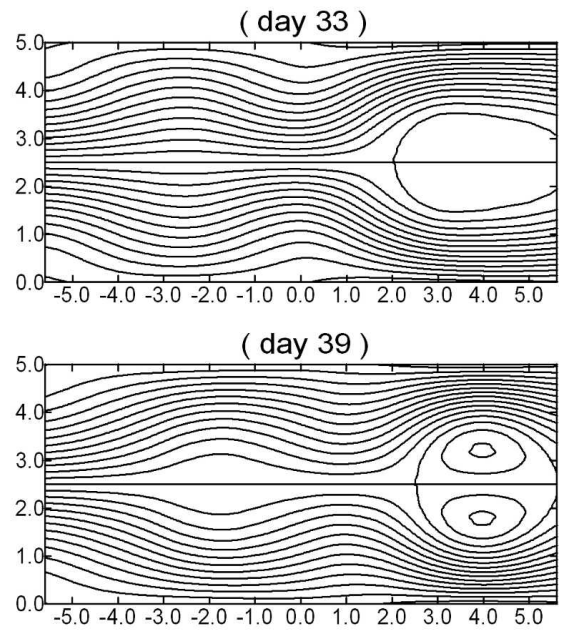

( day 45 )
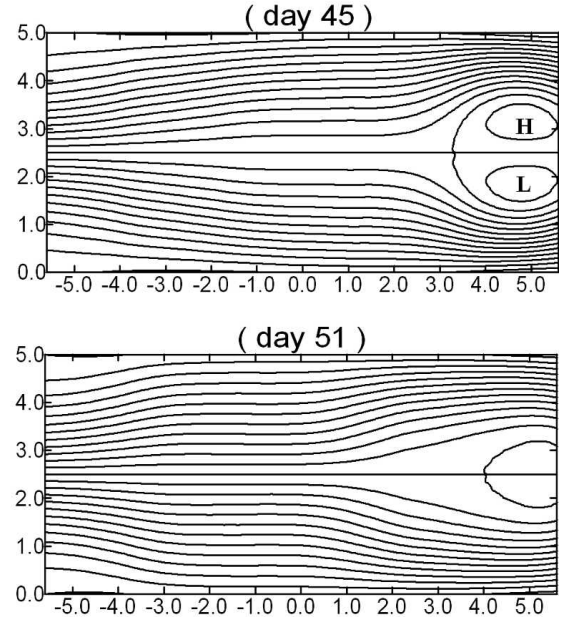

Fig. 5. (Continued)

\section{b. Physical mechanism of positive-phase NAO events}

For a $\mathrm{NAO}^{+}$event, the $F_{e}$ field is found to exhibit a spatial structure opposite to that for the negative-phase event (not shown). Before day 9, upstream synopticscale waves transport continuously cyclonic vorticities into the northern part of the incipient $\mathrm{NAO}^{+}$anomaly and anticyclonic vorticities into the southern part, which makes an incipient $\mathrm{NAO}^{+}$anomaly amplify and eventually forms a jet intensification (low-over-high) anomaly. But then, the reverse process, NAO decay, occurs between day 9 and 15 . Thus, the change of $F_{e}$ in sign and spatial structure controls the growth and decay of an $\mathrm{NAO}^{+}$event. Hurrell (1995) found that the positive NAO phase needs an eddy-forcing pattern opposite to that in Fig. 4. Thus, it can be concluded that whether an $\mathrm{NAO}^{+}\left(\mathrm{NAO}^{-}\right)$event is excited by preex- isting synoptic-scale waves depends crucially upon whether the preexisting planetary-scale wave and the PEF have the matching spatial structure. It must be pointed out that the preexisting planetary wave required by the $\mathrm{NAO}^{+}$event is, in general, stronger than that required by the $\mathrm{NAO}^{-}$event (not shown).

\section{Impact of the NAO on blocking activity over Europe}

It is important to investigate the impact of the NAO on blocking activity over Europe from observations because the theoretical model can show that the breakdown of $\mathrm{NAO}^{+}$events results in the frequent occurrence of downstream blocking.

To demonstrate the applicability of the theoretical results obtained in the above sections, two NAO events (one negative and one positive) are shown in Fig. 6. 
(b)
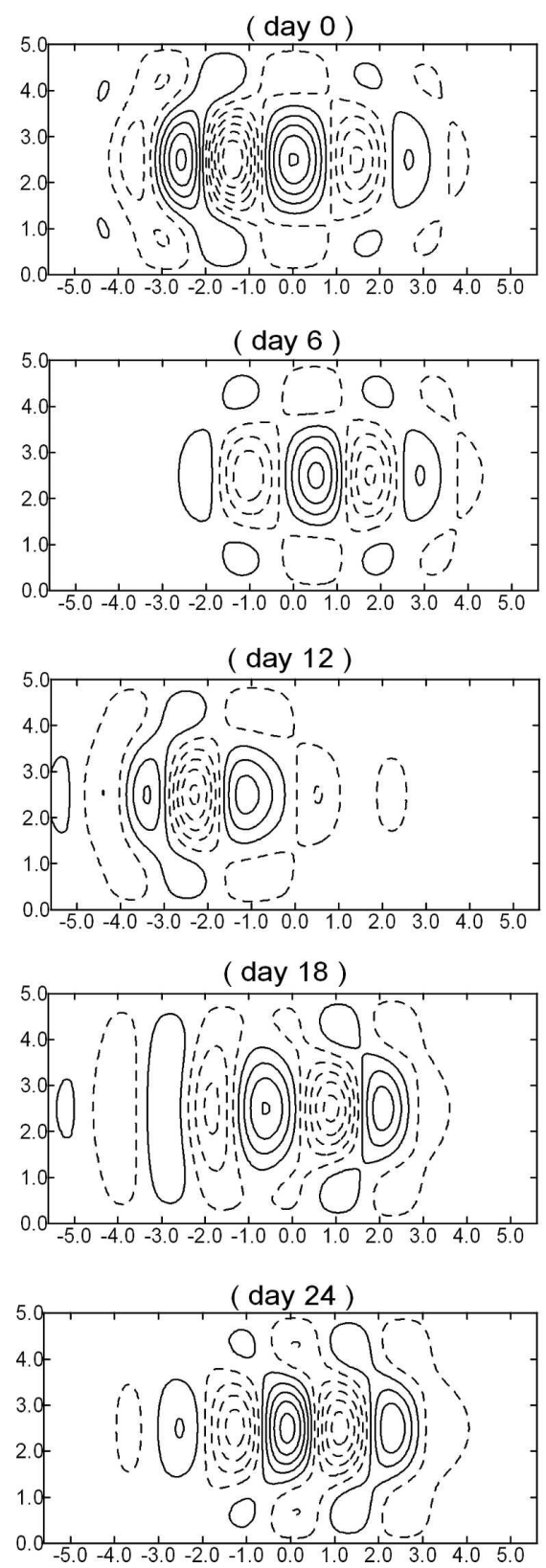
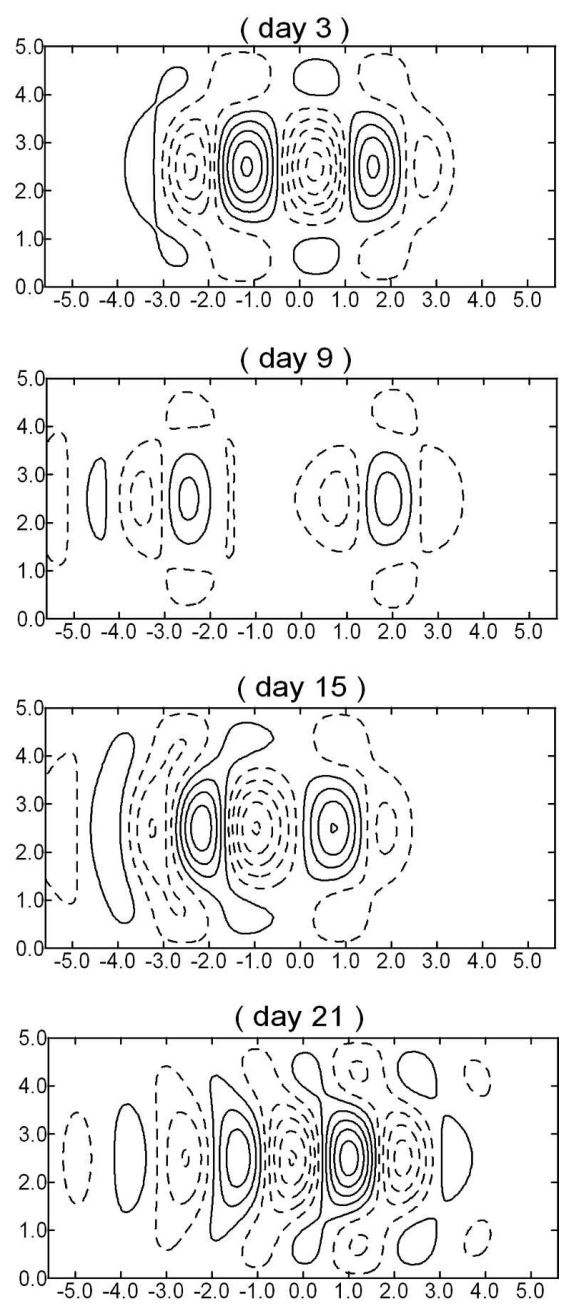

( day 27 )

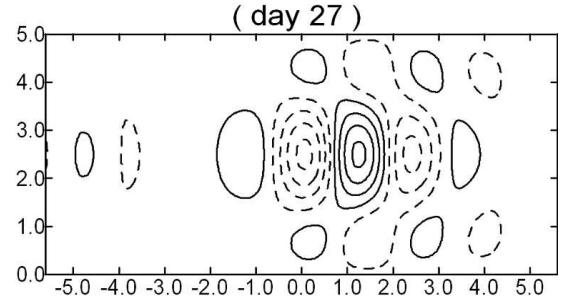

FIG. 5. (Continued)

Figure 6a depicts a $\mathrm{NAO}^{-}$event at $500 \mathrm{hPa}$ from 22 January to 15 February 1965 . The $\mathrm{NAO}^{-}$event is very similar to the life cycle of a blocking flow observed in the Atlantic sector and is also consistent with the theoretical result shown in Fig. 3c. In this case, no blocking events occur over northern Europe. Figure 6b shows the 500-hPa geopotential height fields for a $\mathrm{NAO}^{+}$ event from 19 December 1989 to 7 January 1990. On 25 December 1989, a typical low-over-high dipole similar to a mature $\mathrm{NAO}^{+}$flow is established in the Atlantic sector and then begins to decay. During the decay process of this NAO event, a blocking event occurs downstream (Europe) of the NAO region and completely disappears on 7 January 1990 . Thus, it can be concluded from a comparison with Fig. 5c that the establishment of a blocking event over Europe might be associated with the breakdown of the $\mathrm{NAO}^{+}$event. The life cycle of this positive-phase event looks like the theoretical result shown in Fig. 5c, though their time scales are not completely consistent. Nevertheless, this theoretical re- 


\section{(b) continued}
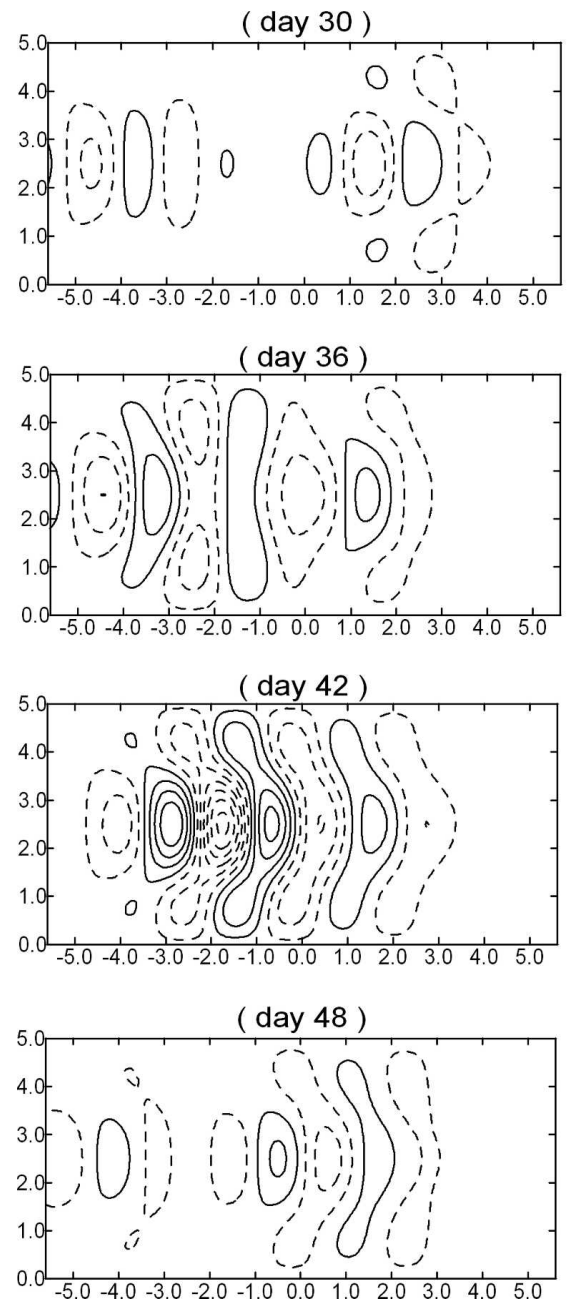
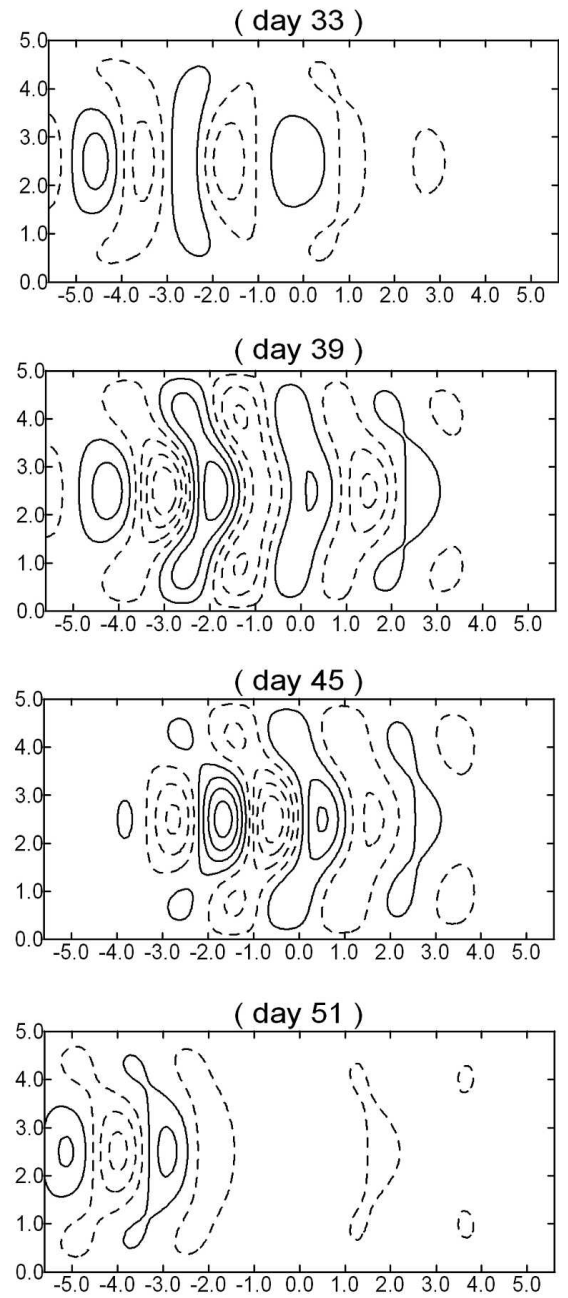

FIG. 5. (Continued)

sult would be more similar to observations if a baroclinic model is used (Luo 2005b).

To further elucidate how the phase of the NAO affects the blocking activity over Europe, winter cases are considered here. A statistical study of blocking events over Europe is performed using the daily mean, 500$\mathrm{hPa}$, gridded $2.5^{\circ}$ latitude $\times 2.5^{\circ}$ longitude reanalysis dataset from the National Centers for Environmental Prediction-National Center for Atmospheric Research (NCEP-NCAR) and using the Lupo and Smith (1995a) definition of blocking events. As noted above, the positive (negative) value of the seasonal mean NAO index can, to some extent, reflect the frequency of $\mathrm{NAO}^{+}$ $\left(\mathrm{NAO}^{-}\right)$events. Thus, it would be helpful to employ the winter mean NAO index to investigate the impact of the NAO on blocking activity over Europe. The negative- and positive-phase years of the strong NAO in winter were taken to be 1956, 1959, 1961, 1965, 1966, 1970, 1978, 1980, $1997\left(\mathrm{NAO}^{-}\right)$and 1974, 1977, 1984, 1985, 1990, 1991, 1994, 1995, $1996\left(\mathrm{NAO}^{+}\right)$, respectively, from the Center for Environmental Prediction Studies at Rutgers University (data available online at http://www.cep.rutgers.edu/ oman/). These $\mathrm{NAO}^{-}$ and $\mathrm{NAO}^{+}$phases are slightly different from the Shabbar et al. classification (Shabbar et al. 2001).

Figure 7 shows the frequency of blocking events over Europe $\left(0^{\circ} \sim 80^{\circ} \mathrm{E}\right)$ for negative and positive phases. It was found that over Europe relatively long-lived blocking events were more frequent during the positive phase than during negative phase. For example, there were six blocking events with periods equal to 10 days or longer during the negative-phase years, but there were 12 cases during the positive-phase years. This shows that the positive phase of the NAO seems to 
(c)
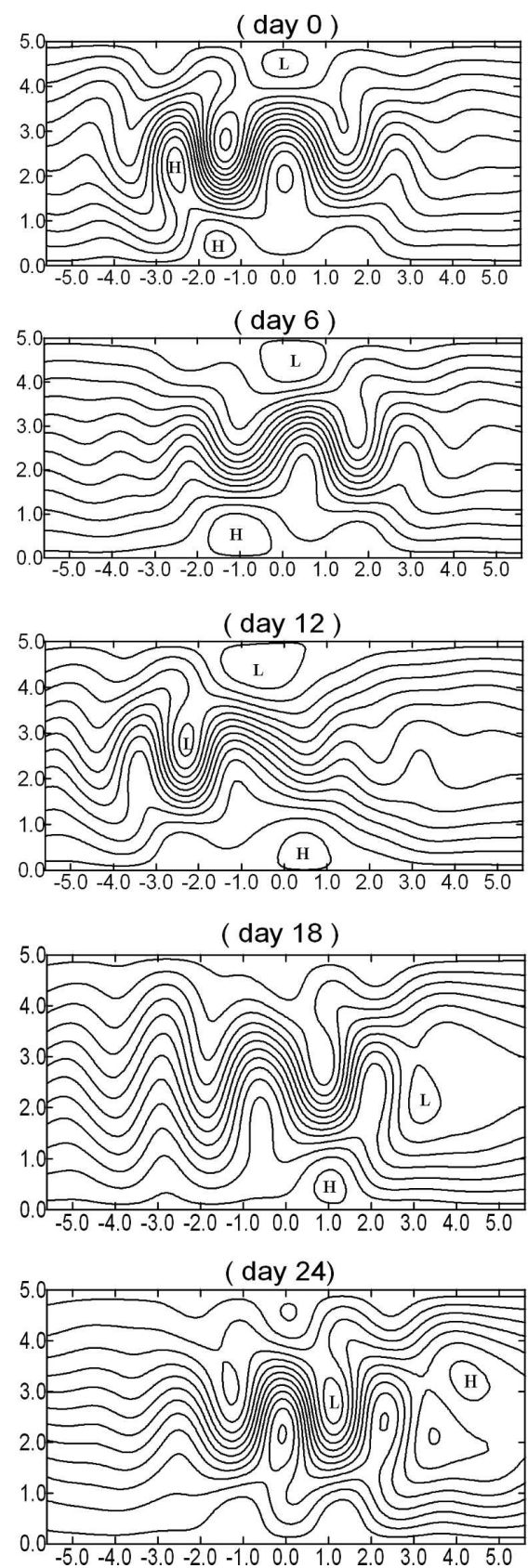

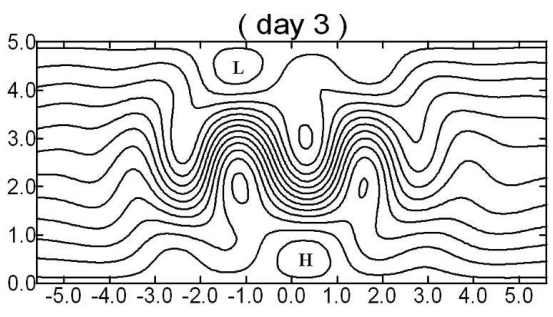

( day 9 )
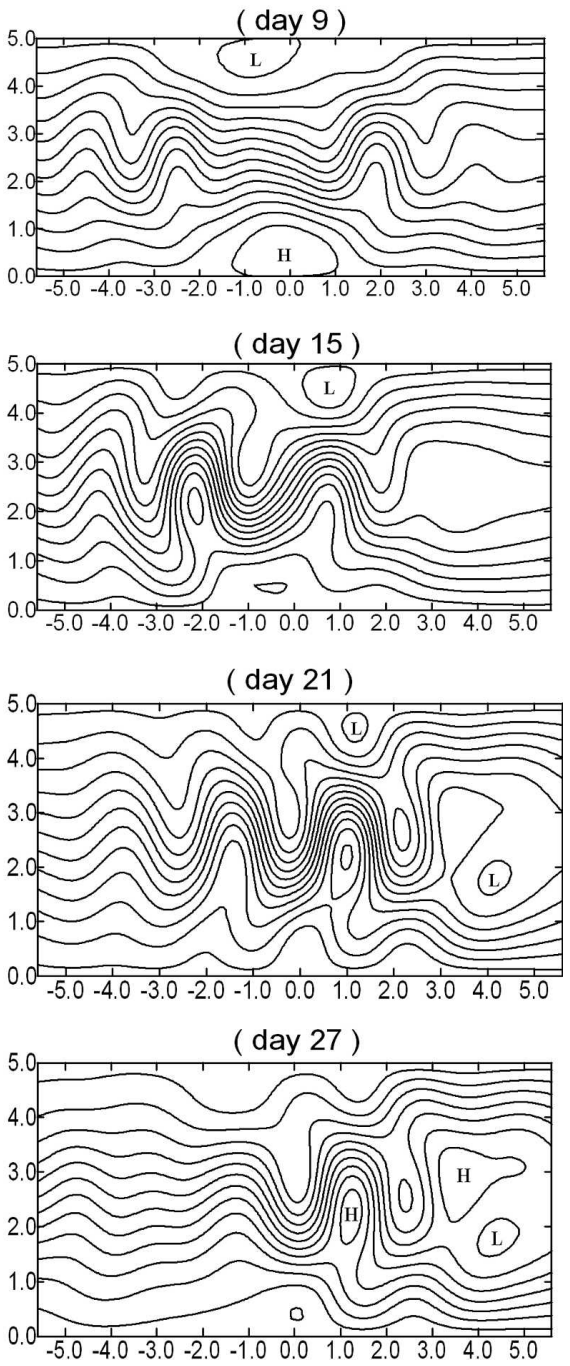

FIG. 5. (Continued) favor the occurrence of long-lived blocking events over Europe. In addition, there were more blocking events and blocking days during the $\mathrm{NAO}^{+}$phase than during the negative phase, as shown in Fig. 8. Thus, the $\mathrm{NAO}^{+}$ phase also favored the frequent occurrence of blocking events over Europe, which is a confirmation of our theoretical result. In the numerical experiment of Franzke et al. (2004), blocking was also found to occur over Europe after the decay of a $\mathrm{NAO}^{+}$event (their Fig. 5).
Although the physical mechanism responsible for the $\mathrm{NAO}^{+}$favoring the onset of European blockings was not explored in this paper, the radiation of Rossby waves (energy dispersion of Rossby waves) within the NAO region is implied to be the cause of the frequent occurrence of blocking events over Europe. This issue deserves further study. Even so, our theoretical model demonstrates that the positive phase of the NAO is able to promote the occurrence of European blocking 


\section{(c) continued}
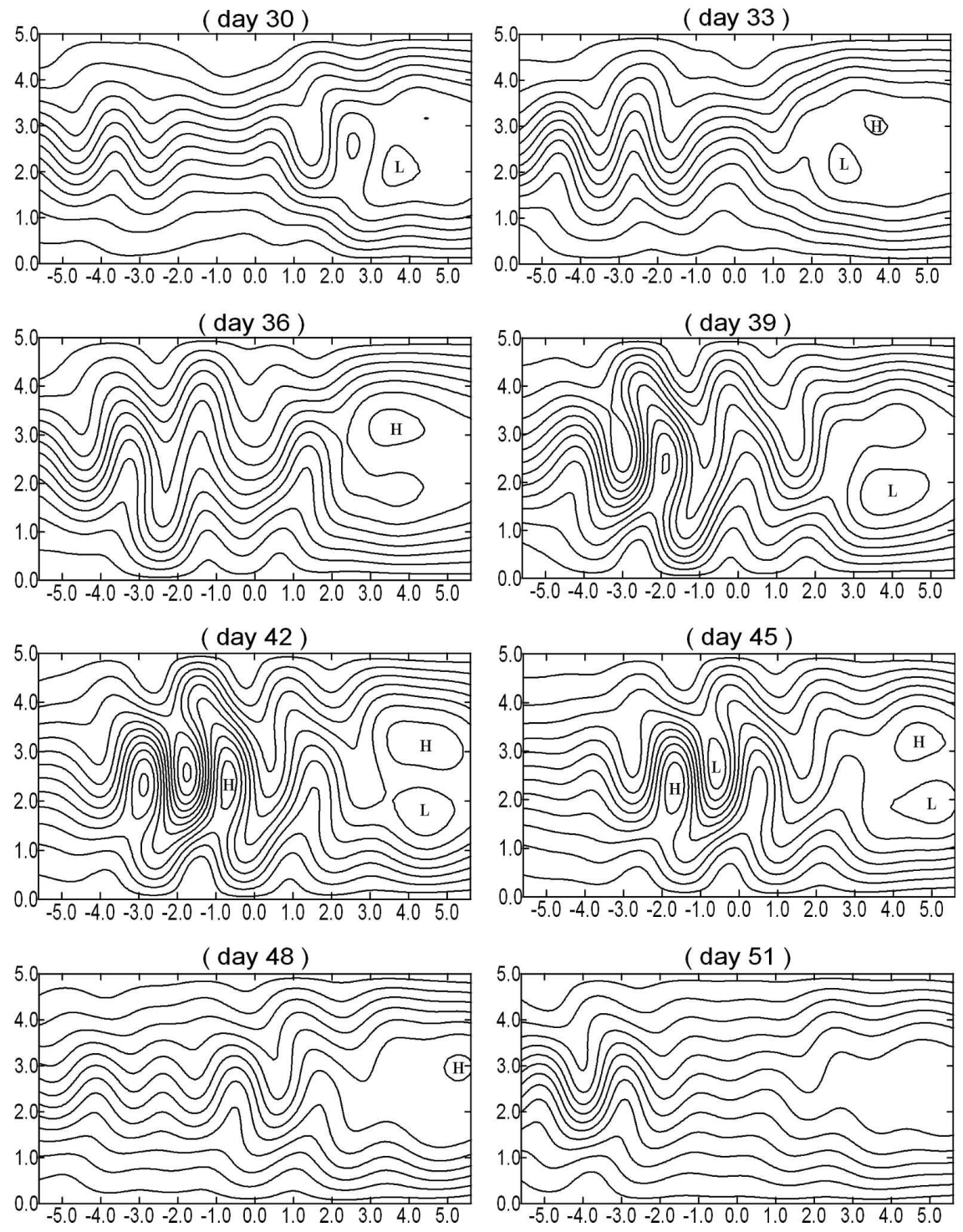

FIG. 5. (Continued) events, which is not found in previous theoretical NAO models.

\section{Relationship between the NAO and the zonal index}

In previous studies of the NAO and annular modes, the first empirical orthogonal function of the zonal mean wind is usually referred to as the zonal index (ZI), but most atmospheric dynamicists regard the ZI to be the zonal mean of the NAO and annular mode (Feldstein 2003). We will concentrate here on exploring similarities between the dynamical processes that account for the growth, maintenance, and decay of the ZI and that of the NAO (Feldstein 2003).

Using (9), the modified zonal mean flow, due to the feedback (vorticity flux) of the NAO anomaly, can be defined as

$$
\begin{aligned}
u_{m} & =u_{0}+u_{a}, \\
u_{a} & =-\varepsilon^{2} \frac{\partial \tilde{\psi}_{1}}{\partial y} \\
& =-|B|^{2} \sum_{n=1}^{\infty} q_{n} g_{n}(n+1 / 2) m \sin [(n+1 / 2) m y] .
\end{aligned}
$$


(a)
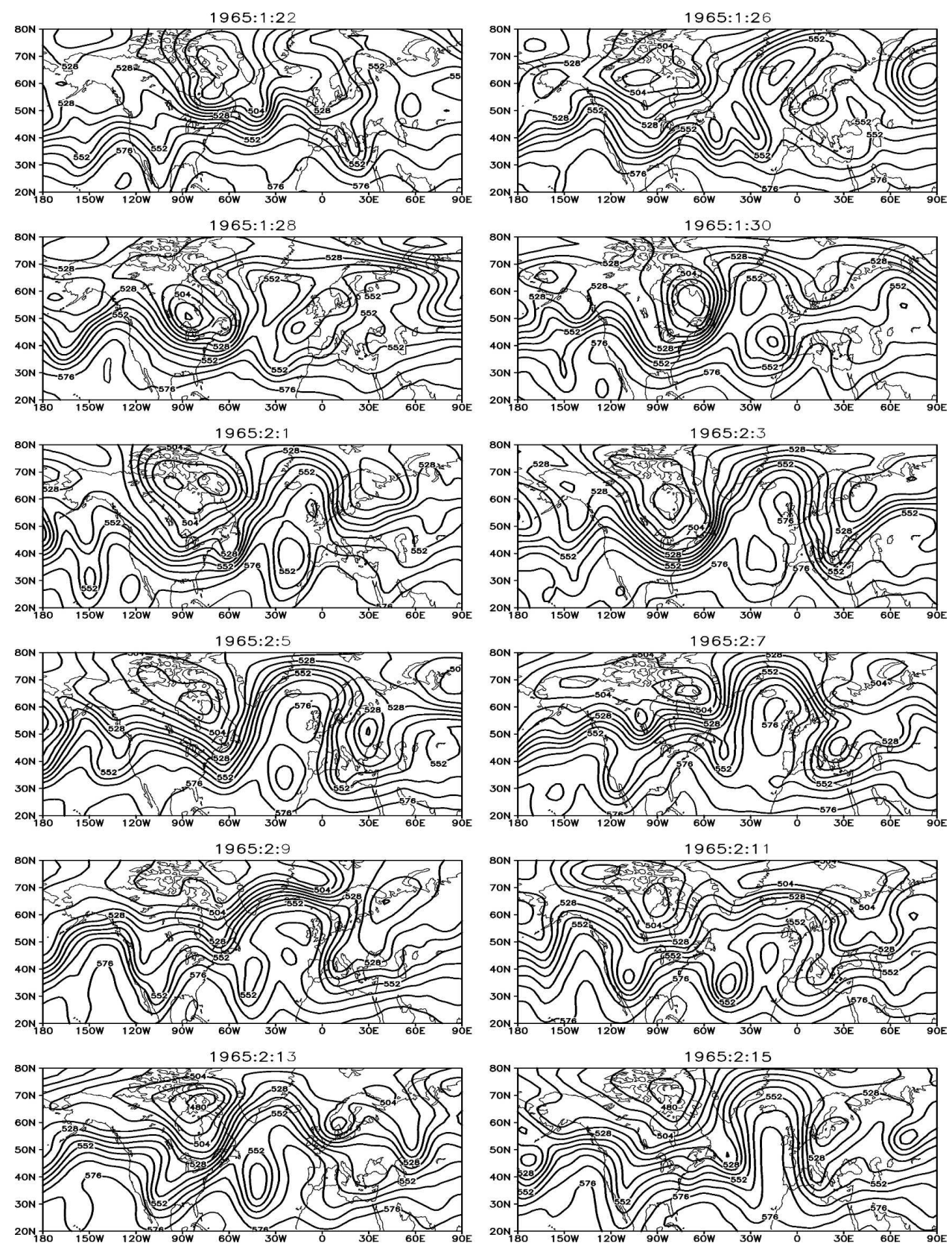

FIG. 6. Geopotential height fields $(10 \mathrm{gpm})$ at $500 \mathrm{hPa}$ for negative- and positive-phase NAO events occurring during the periods from 22 Jan to 15 Feb 1965 and from 19 Dec 1989 to 7 Jan 1990, respectively (contour interval is 8). (a) Negative phase and (b) positive phase.

It is easy to find from (10b) that the westerly anomaly $u_{a}$ depends largely upon the amplitude of the NAO. The anomaly is, however, independent of the phase of the NAO because $u_{a}$ does not depend on the sign of $m$, which determines the phase of the NAO. Since the zonal index (ZI) is a zonal mean quantity, $u_{a}$ can be used to represent the ZI during NAO episodes al- though $A$ is only a slowly varying quantity in zonal space and time.

The Lejenas and Okland (1983) blocking index is also proportional to the $\mathrm{ZI}$; thus we can examine the results in this section as a correspondence between indicators of blocking events and NAO events. It can be anticipated from (10b) that there is a high correlation 
(b)
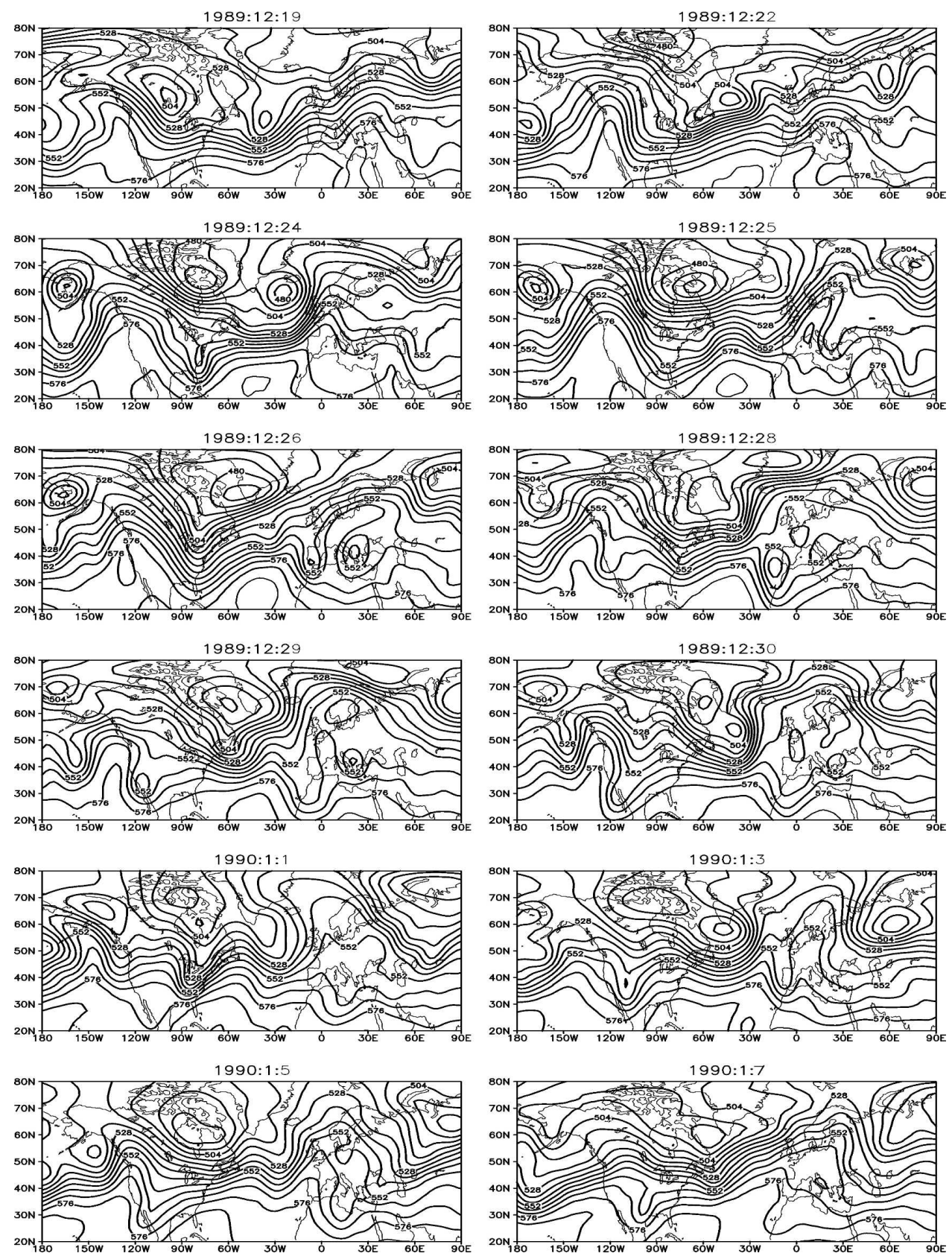

Fig. 6. (Continued)

between the amplitude of ZI and the NAO index time series (DeWeaver and Nigam 2000). This also explains why there are many similarities between the dynamics of the ZI and NAO (Feldstein 2003).

On the other hand, we find using (10b) that the ZI and the NAO have the same lifetime because the NAO anomaly amplitude $A$ is included in the variation of the zonal flow. Thus, it is inevitable that the ZI completes its cycle of growth and decay within approximately two weeks, consistent with that of NAO. Since the NAO is driven by high-frequency transient eddy fluxes, the ZI is inevitably also driven by the high-frequency transient eddy fluxes. These theoretical findings are in agreement with numerical results and observations in previous studies (Robinson 1991, 1996; Yu and Hartman 1993; Feldstein and Lee 1998; Limpasuvan and Hart- 
(a)

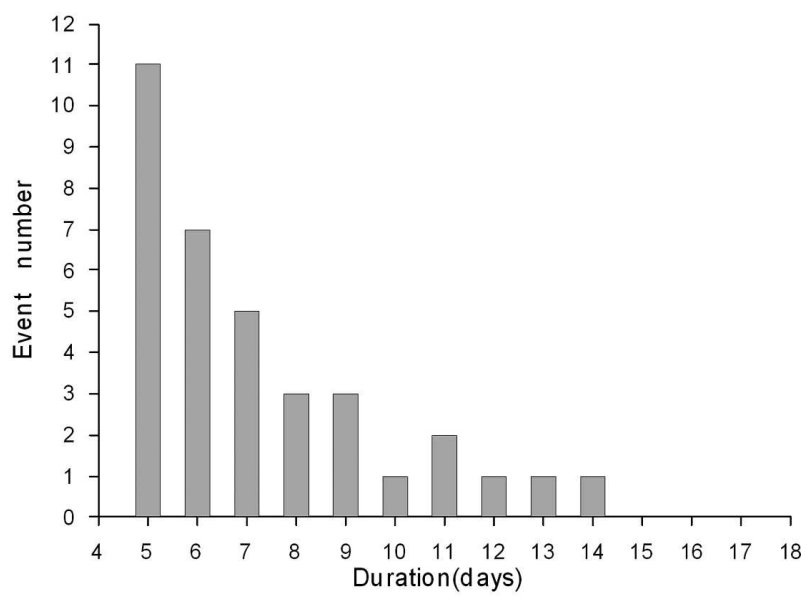

(b)

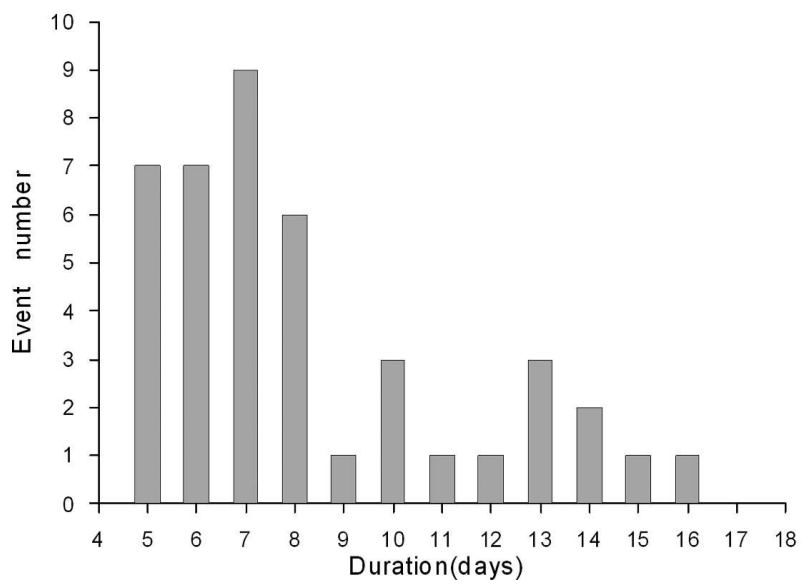

FIG. 7. Frequency and duration of blocking events over Europe for two phases of the NAO: (a) negative phase and (b) positive phase.

man 2000; Wallace 2000; Feldstein 2003). Although numerical modeling and diagnostic studies have confirmed this relationship, an analytical solution for a relationship between the ZI and NAO was not derived theoretically. This problem can be understood from (10b).

Figure 9 shows the instantaneous field of the westerly anomaly $u_{a}$ for the same parameters as in Fig. 5. During the life cycle of a $\mathrm{NAO}^{+}$we found that (from day 0 to $21)$ the negative anomalies exist at middle latitudes, but the positive anomalies appear at high and low latitudes, respectively. This implies that the eddy forcing accelerates the zonal mean westerly wind at both high and low latitudes but decelerates the westerly wind at middle latitudes through the excitation of a NAO anomaly. This result is consistent with the numerical results of Robinson (1991) and Yu and Hartman (1993), who (a)

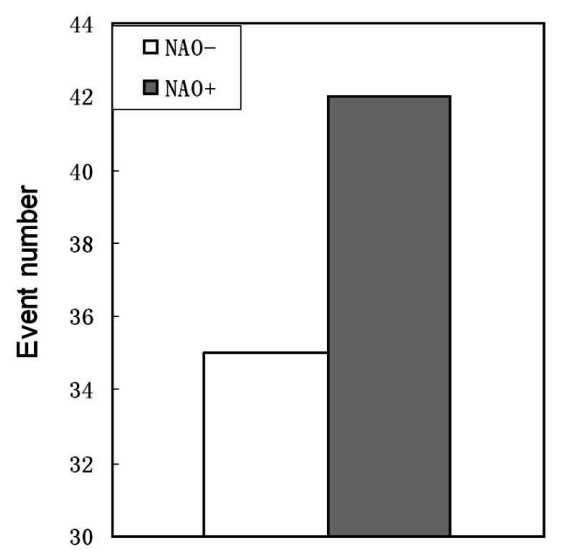

(b)

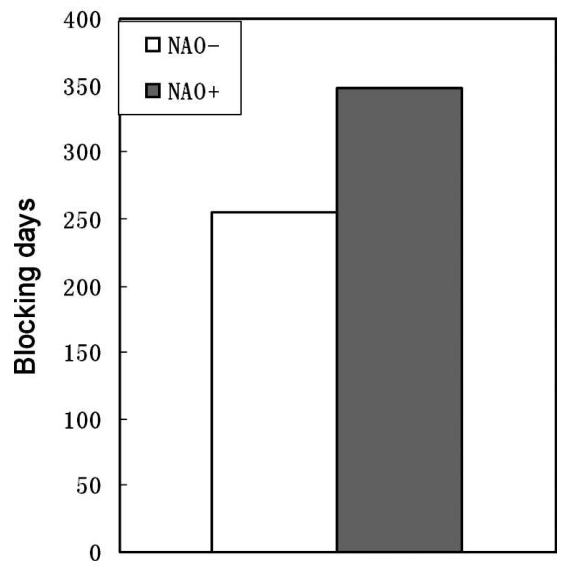

FIG. 8. Number of blocking events and blocking days over Europe for two phases of the NAO: (a) blocking events and (b) blocking days.

found that barotropic eddy forcing sustains the zonal flow anomalies and drives the zonal flow vacillation. Thus, the ZI and the NAO can be driven simultaneously by synoptic-scale waves. Because many physical processes such as land-sea contrast topography are ignored in this paper, the zonal flow anomalies induced by high-frequency transient eddies cannot be completely consistent with observations. Thus, the theoretical results obtained in this paper should be extended to a baroclinic model and include other factors that possibly affect observed westerly anomalies.

It must be pointed out that the zonal mean wind anomaly in Fig. 9 is independent of whether the NAO is in a high-over-low configuration or a low-over-high due to the exclusion of large-scale land-sea contrast topography in the NH in our theoretical model. As the NH land-sea contrast topography is included, the zonal wind anomaly will be strongly dependent upon the phase of the NAO event in that climatological station- 
( day 0 )

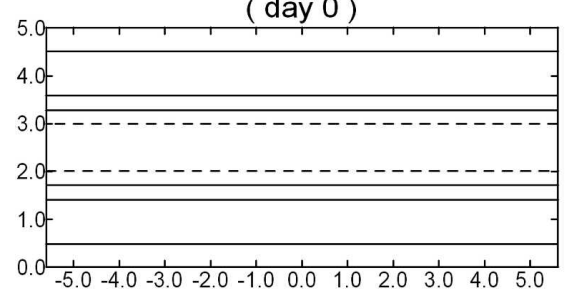

( day 6 )

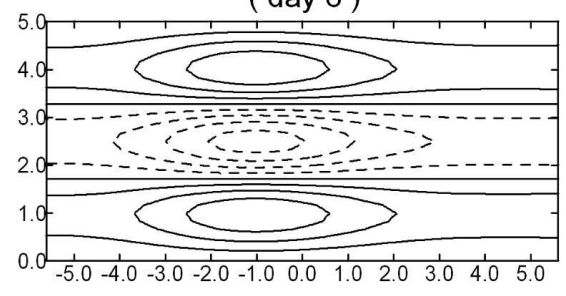

( day 12 )

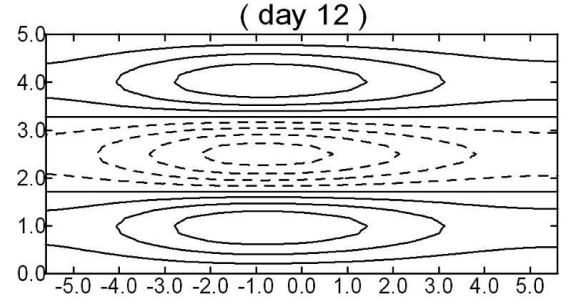

( day 18 )

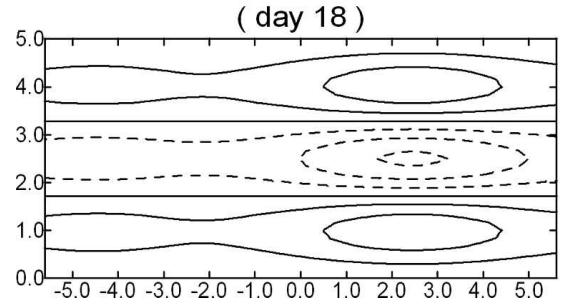

( day 3 )

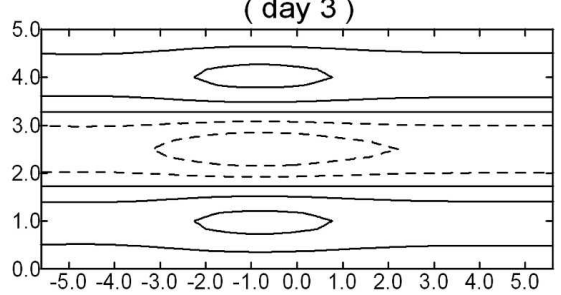

( day 9 )

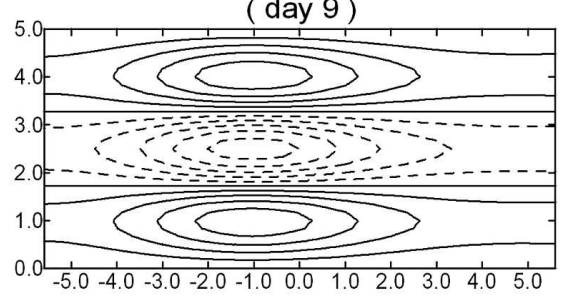

( day 15 )

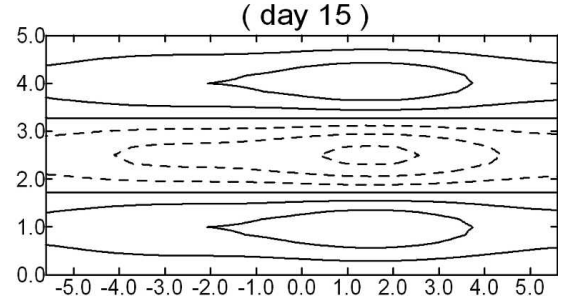

( day 21 )

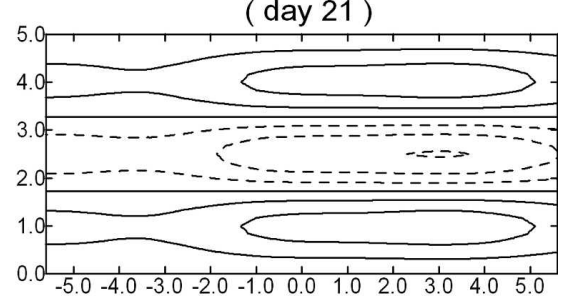

FIG. 9. Evolution of westerly anomaly $u_{a}$ during the positive NAO phase for the same parameters as in Fig. $5(\mathrm{CI}=0.1)$, in which the dashed curve denotes the negative westerly anomaly and the solid curve denotes the positive westerly anomaly.

ary waves in the Northern Hemisphere can be excited by the land-sea contrast topography (not shown). As found by DeWeaver and Nigam (2000), the interaction between transient eddies (low frequency) and climatological stationary waves plays a large role in the ZI life cycle associated with the NAO. Thus, it is necessary that the NAO model presented here be extended to include land-sea contrast topography in order to simulate exactly the life cycle of the ZI. This deserves further study.

On the other hand, if the $\mathrm{ZI}$ is defined as a regional mean of the NAO, the zonal mean wind anomaly is affirmatively dependent on the phase of the NAO because such a regional mean cannot exactly remove the components (wavenumber $k$ ) of the NAO. In our weakly nonlinear model, when the climatological stationary waves induced by external forcing, such as the land-sea contrast topography, are absent, the ZI is naturally independent of the phase of the NAO because the NAO anomaly in (9a) is meridionally antisymmetric.

\section{Conclusions and discussion}

We have extended here the block-eddy interaction model proposed in Luo (2005a) to examine the dynamical mechanism of the life cycle of the NAO driven by synoptic-scale waves. This theoretical model not only described the evolution of the NAO in its two phases, but also clarifies what determines the phase of the NAO. It was shown that onset of the NAO can be interpreted as a nonlinear initial-value problem. The onset and decay of the NAO within a time period of two weeks is essentially due to a change in the preexisting eddy forcing (PEF) (high-frequency eddy fluxes) from synoptic-scale waves. The PEF is found to play either a positive role that drives the NAO to grow dur- 
ing the onset phase or a negative role that drives the NAO to decay during the decay phase. The positive and negative feedbacks of the PEF form the essence of the NAO life cycle in which the negative feedback is similar to the role played by Ekman pumping in the NAO decay noted by Feldstein (2003). Whether a $\mathrm{NAO}^{-}$event occurs depends primarily upon if both the planetary-scale flow prior to the NAO event and the PEF have matching spatial structure. When the preexisting planetary-scale flow with a high-over-low structure matches the PEF field with a negative-overpositive structure, the preexisting synoptic-scale waves can interact with the preexisting planetary-scale flow to excite a $\mathrm{NAO}^{-}$event. $\mathrm{A} \mathrm{NAO}^{+}$event can be excited if the preexisting planetary-scale flow has a low-over-high structure and if the initial PEF field has a positive-overnegative structure. This provides a reasonable answer for what process determines the phase of the NAO (Benedict et al. 2004).

An important finding in this paper is that $\mathrm{NAO}^{-}$ events occurred repeatedly within the NAO region after the initial $\mathrm{NAO}^{-}$event has decayed, but for the $\mathrm{NAO}^{+}$events isolated dipole blocks can occur downstream of the $\mathrm{NAO}$ region after the initial $\mathrm{NAO}^{+}$event has decayed. This implies that the frequency of European blocks is higher during the positive NAO phase than during the negative NAO phase (Shabbar et al. 2001; Barriopedro et al. 2006). This result is confirmed further using the statistical study presented in section 6 . Additionally, it was shown that during the positive NAO phase isolated blocks over Europe were longlived and frequent, indicating that the breakdown of the $\mathrm{NAO}^{+}$events can result in the frequent occurrence of European blocking.

Furthermore, it is demonstrated here that the life cycle of the ZI is dominated by a change in the amplitude of the NAO, indicating that both the ZI and the NAO have the same dynamics. The PEF tends to excite a zonal mean wind anomaly through the self-interaction of the NAO anomaly, in which the zonal mean westerly winds are accelerated at both high and low latitudes but decelerated at middle latitudes during the NAO life cycle.

However, it should be pointed out that the zonal mean wind anomalies shown in Fig. 9 are independent of the phase of the NAO. This may be due to the lack of land-sea contrast topography in our theoretical model since the climatological stationary waves in the Northern Hemisphere are usually induced by the largescale land-sea contrast topography and play a large role in driving the zonal mean wind anomalies associated with the NAO (DeWeaver and Nigam 2000).

Although the results of this paper can capture the main features of the NAO life cycle, they seem to be unable to explain better why the $\mathrm{NAO}^{+}$phase is preceded by a low-frequency wave train over the North Pacific, whereas the $\mathrm{NAO}^{-}$phase develops in situ (Feldstein 2003; Benedict et al. 2004). Even so, the theoretical model proposed in the present study can be applied to the dynamics of eddy-driven low-frequency dipole modes such as the Northern Hemispheric annular mode (NAO/AO) or Southern Hemispheric annular mode (Antarctic Oscillation) because the PEF from preexisting synoptic waves having a monopole structure exhibits a dipole meridional structure. Of course, this model may be also applicable to the PNA pattern when the synoptic-scale eddies consist of monopole and dipole meridional structures, which is beyond the scope of this work. Although this study provides some answers to the main questions proposed by Benedict et al. (2004), the exact role of atmospheric baroclinic, stratospheric, and oceanic processes play in the NAO life cycle is still unresolved. These topics need further research. In Part II of this paper (Luo et al. 2007), we will confirm with diagnostic studies of NAO cases and composite fields that the NAO events can be approximated as free modes.

Acknowledgments. The first and third authors acknowledge the support from the National Outstanding Youth Natural Science Foundation of China under Grant 40325016 and the National Natural Science Foundation of China (Grant 4057016), National Basic Research Program (Program 973) (2005CB422302), and FANEDD. The second author would also like to thank the support of the Missouri Research Board and U.S. Department of Energy. We are grateful to Dr. Yina Diao for her help during this study and also gratefully acknowledge discussions with Profs. Zhengyu Liu, Bin Wang, Lixin Wu, and Shanping Xie. The authors thank Dr. S. Feldstein and an anonymous reviewer for useful suggestions in improving this paper.

\section{APPENDIX}

\section{Coefficients Used in Eq. (7)}

$$
p_{j}=\frac{\left(k-2 \tilde{k}_{j}\right)}{\left(\beta+F u_{0}\right)\left(\tilde{k}_{j}+k\right)-\left[u_{0}\left(\tilde{k}_{j}+k\right)-\left(\tilde{\omega}_{j}+\omega\right)\right]\left[\left(\tilde{k}_{j}+k\right)^{2}+9 m^{2} / 4+F\right]},
$$




$$
\begin{aligned}
& r_{j}=\frac{\left(k+2 \tilde{k}_{j}\right)}{\left(\beta+F u_{0}\right)\left(\tilde{k}_{j}+k\right)-\left[u_{0}\left(\tilde{k}_{j}+k\right)-\left(\tilde{\omega}_{j}+\omega\right)\right]\left[\left(\tilde{k}_{j}+k\right)^{2}+m^{2} / 4+F\right]}, \\
& s_{j}=\frac{\left(k+2 \tilde{k}_{j}\right)}{\left(\beta+F u_{0}\right)\left(\tilde{k}_{j}-k\right)-\left[u_{0}\left(\tilde{k}_{j}-k\right)-\left(\tilde{\omega}_{j}-\omega\right)\right]\left[\left(\tilde{k}_{j}-k\right)^{2}+9 m^{2} / 4+F\right]}, \\
& h_{j}=\frac{\left(k-2 \tilde{k}_{j}\right)}{\left(\beta+F u_{0}\right)\left(\tilde{k}_{j}-k\right)-\left[u_{0}\left(\tilde{k}_{j}-k\right)-\left(\tilde{\omega}_{j}-\omega\right)\right]\left[\left(\tilde{k}_{j}-k\right)^{2}+m^{2} / 4+F\right]} \\
& (j=1,2) .
\end{aligned}
$$

\section{REFERENCES}

Barriopedro, D., R. Garcia-Herrera, A. R. Lupo, and E. Hernandez, 2006: A climatology of Northern Hemisphere blocking. J. Climate, 19, 1042-1063.

Benedict, J. J., S. Lee, and S. B. Feldstein, 2004: Synoptic view of the North Atlantic Oscillation. J. Atmos. Sci., 61, 121-144.

Berggren, R., B. Bolin, and C. G. Rossby, 1949: An aerological study of zonal motion, its perturbations and break-down. Tellus, 1, 14-37.

Christoph, M., U. Ulbrich, J. M. Oberhuber, and E. Roeckner, 2000: The role of ocean dynamics for low-frequency fluctuations of the NAO in a coupled ocean-atmosphere GCM. $J$. Climate, 13, 2536-2549.

DeWeaver, E., and S. Nigam, 2000: Do stationary waves drive the zonal-mean jet anomalies of the northern winter? J. Climate, 13, 2160-2176.

Feldstein, S. B., 2003: The dynamics of NAO teleconnection pattern growth and decay. Quart. J. Roy. Meteor. Soc., 129, 901924.

— an eddy feedback? J. Atmos. Sci., 55, 3077-3086.

Franzke, C., S. Lee, and S. B. Feldstein, 2004: Is the North Atlantic Oscillation a breaking wave? J. Atmos. Sci., 61, 145160 .

Frederiksen, J. S., 1982: A unified three-dimensional instability theory of the onset of blocking and cyclogenesis. J. Atmos. Sci., 39, 969-982.

Gerber, E. P., and G. K. Vallis, 2005: A stochastic model for the spatial structure of annular patterns of variability and the North Atlantic Oscillation. J. Climate, 18, 2102-2118.

Haines, K., and P. Malanotte-Rizzoli, 1991: Isolated anomalies in westerly jet streams: A unified approach. J. Atmos. Sci., 48, $510-526$.

Hurrell, J. W., 1995: Transient eddy forcing of the rotational flow during northern winter. J. Atmos. Sci., 52, 2286-2301.

Jin, F. F., L. L. Pan, and M. Watanabe, 2006a: Dynamics of synoptic eddy and low-frequency flow interaction. Part I: A linear closure. J. Atmos. Sci., 63, 1677-1694.

,-- , and $\longrightarrow, 2006 \mathrm{~b}$ : Dynamics of synoptic eddy and lowfrequency flow interaction. Part II: A theory for lowfrequency modes. J. Atmos. Sci., 63, 1695-1708.

Lejenas, H., and H. Okland, 1983: Characteristics of Northern Hemisphere blocking as determined from a long time series of observational data. Tellus, 35A, 350-362.

Limpasuvan, V., and D. L. Hartmann, 2000: Wave-maintained annular modes of climate variability. J. Climate, 13, 4414-4429.

Luo, D., 2005a: A barotropic envelope Rossby soliton model for block-eddy interaction. Part I: Effect of topography. J. Atmos. Sci., 62, 5-21.

_ 2005b: Interaction between envelope soliton vortex pair blocks and synoptic-scale eddies in an inhomogeneous baroclinicity environment. Quart. J. Roy. Meteor. Soc., 131, 125154.

— F. Fuang, and Y. Diao, 2001: Interaction between antecedent planetary-scale envelope soliton blocking anticyclone and synoptic-scale eddies: Observations and theory. J. Geophys. Res., 106, 31 795-31 816.

— low-frequency dipole modes. Part II: Free mode characteristics of NAO and diagnostic study. J. Atmos. Sci., 64, 29-51.

Lupo, A. R., and P. J. Smith, 1995a: Climatological features of blocking anticyclones in the Northern Hemisphere. Tellus, 47A, 439-456.

$\longrightarrow$, and $\longrightarrow$, 1995b: Planetary and synoptic-scale interactions during the life cycle of a mid-latitude blocking anticyclone over the North Atlantic. Tellus, 47A, 575-596.

Malguzzi, P., and P. Malanotte-Rizzoli, 1984: Nonlinear stationary Rossby waves on non-uniform zonal winds and atmospheric blocking: The analytical theory. J. Atmos. Sci., 41, 2620-2628.

Marshall, J., H. Johnson, and J. Goodman, 2001: A study of the interaction of the North Atlantic Oscillation with ocean circulation. J. Climate, 14, 1399-1422.

Nakamura, H., and J. M. Wallace, 1993: Synoptic behavior of baroclinic eddies during the blocking onset. Mon. Wea. Rev., 121, 1892-190.

Quadrelli, R., M. Lazzeri, C. Cacciamani, and S. Tibaldi, 2001: Observed winter Alpine precipitation variability and links with large-scale circulation patterns. Climate Res., 17, 275284.

Robinson, W. A., 1991: The dynamics of the zonal index in a simple model of the atmosphere. Tellus, 43A, 295-305.

- 1996: Does eddy feedback sustain variability in the zonal index? J. Atmos. Sci., 53, 3556-3569.

Scherrer, S. C., C. Schwierz, and C. Appenzellor, 2006: Twodimensional indices of atmospheric blocking and their statistical relationship with winter climate patterns in EuroAtlantic region. Int. J. Climatol., 26, 233-249.

Shabbar, A., J. Huang, and K. Higuchi, 2001: The relationship between the wintertime North Atlantic Oscillation and blocking episodes in the North Atlantic. Int. J. Climatol., 21, 355-369.

Shutts, G. J., 1983: The propagation of eddies in diffluent jet streams: Eddy vorticity forcing of blocking flow fields. Quart. J. Roy. Meteor. Soc., 109, 737-761. 
, 1986: A case study of eddy forcing during an Atlantic blocking episode. Advances in Geophysics, Vol. 29, Academic Press, 135-161.

Thompson, D. W. J., and J. M. Wallace, 2000: Annular modes in the extratropical circulation. Part I: Month-to-month variability. J. Climate, 13, 1000-1016.

,-- , and G. C. Hegerl, 2000: Annular modes in the extratropical circulation. Part II: Trends. J. Climate, 13, 1018-1036.

Vallis, G. K., E. P. Gerber, P. J. Kushner, and B. A. Cash, 2004: A mechanism and simple dynamical model of the North Atlantic Oscillation and annular modes. J. Atmos. Sci., 61, 264-280.

Visbeck, M. H., J. W. Hurrell, L. Polvani, and H. M. Cullen, 2001:
The North Atlantic oscillation: Past, present and future. Proc. Natl. Acad. Sci. USA, 98, 12 876-12 877.

Wallace, J. M., 2000: North Atlantic Oscillation/annular mode: Two paradigms-One phenomenon. Quart. J. Roy. Meteor. Soc., 126, 791-805.

Wu, L., and Z. Liu, 2005: North Atlantic decadal variability: Airsea coupling, oceanic memory, and potential Northern Hemisphere resonance. J. Climate, 18, 331-349.

$\mathrm{Wu}, \mathrm{P}$., and C. Gordon, 2002: Oceanic influence on North Atlantic climate variability. J. Climate, 15, 1911-1926.

Yu, J. Y., and D. L. Hartman, 1993: Zonal flow vacillation and eddy forcing in a simple GCM of the atmosphere. J. Atmos. Sci., 50, 3244-3259. 